\title{
Zeroizing Without Low-Level Zeroes: New MMAP Attacks and Their Limitations ${ }^{1}$
}

\author{
Jean-Sébastien Coron ${ }^{2} \quad$ Craig Gentry ${ }^{3} \quad$ Shai Halevi $^{3} \quad$ Tancrède Lepoint $^{4}$ \\ Hemanta K. Maji ${ }^{5,6} \quad$ Eric Miles $^{6} \quad$ Mariana Raykova $^{7} \quad$ Amit Sahai $^{6,8}$ \\ Mehdi Tibouchi ${ }^{9}$
}

June 15, 2015

\begin{abstract}
We extend the recent zeroizing attacks of Cheon, Han, Lee, Ryu and Stehlé (Eurocrypt'15) on multilinear maps to settings where no encodings of zero below the maximal level are available. Some of the new attacks apply to the CLT13 scheme (resulting in a total break) while others apply to (a variant of) the GGH13 scheme (resulting in a weak-DL attack). We also note the limits of these zeroizing attacks.
\end{abstract}

Keywords: Cryptanalysis, Hardness Assumptions, Multilinear Maps.

\footnotetext{
${ }^{1}$ This work subsumes and extends the previous works [13, 8].

${ }^{2}$ University of Luxembourg. jean-sebastien. coron@uni.lu

${ }^{3}$ IBM Research

${ }^{4}$ CryptoExperts. tancrede.lepoint@cryptoexperts.com. This work has been supported in part by the European Union's H2020 Programme under grant agreement number ICT-644209.

${ }^{5}$ Purdue University

${ }^{6}$ University of California, Los Angeles and Center for Encrypted Functionalities. hemanta.maji@gmail.com, enmiles@cs.ucla.edu, amitsahai@gmail.com

${ }^{7}$ SRI International. mariana@cs. columbia.edu. This work has been supported in part from NSF Award 1421102.

${ }^{8}$ Research supported in part from a DARPA/ONR PROCEED award, a DARPA/ARL SAFEWARE award, NSF Frontier Award 1413955, NSF grants 1228984, 1136174, 1118096, and 1065276, a Xerox Faculty Research Award, a Google Faculty Research Award, an equipment grant from Intel, and an Okawa Foundation Research Grant. This material is based upon work supported by the Defense Advanced Research Projects Agency through the U.S. Office of Naval Research under Contract N00014-11-1-0389. The views expressed are those of the author and do not reflect the official policy or position of the Department of Defense, the National Science Foundation, or the U.S. Government.

${ }^{9}$ NTT Secure Platform Laboratories. tibouchi.mehdi@lab.ntt.co.jp
} 


\section{Contents}

\begin{tabular}{llr}
\hline & Introduction & 1
\end{tabular}

1.1 Impact of Our Attacks . . . . . . . . . . . . . . . . . . . . 1

1.2 Limitations of Zeroizing attacks $\ldots \ldots \ldots \ldots \ldots \ldots \ldots$

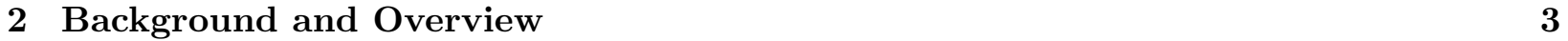

2.1 A Brief Description of the GGH13 and CLT13 Schemes . . . . . . . . . . . . . 3

2.1 .1 The GGH13 scheme . . . . . . . . . . . . . . . . . . . 3

2.1 .2 The CLT13 Scheme . . . . . . . . . . . . . . . . . . . . . 4

2.1 .3 Common Properties . . . . . . . . . . . . . . . . . . . . 4

2.2 Overview of Existing Attacks $\ldots \ldots \ldots \ldots \ldots \ldots$

2.3 Extending the CHLRS Attack $\ldots \ldots \ldots \ldots \ldots \ldots \ldots$

2.3 .1 GGH13 vs. CLT13 . . . . . . . . . . . . . . . . . . . . 6

2.3 .2 Orthogonal encodings . . . . . . . . . . . . . . . . . 7

2.3 .3 More than one monomial . . . . . . . . . . . . . . . . . . . 7

2.3 .4 Using Cayley-Hamilton . . . . . . . . . . . . . . . . . . . 7

2.4 Attack Limitations $\ldots \ldots \ldots \ldots \ldots$

3 A Unified Attack against CLT13-Based Schemes 9

3.1 Sufficient conditions for the attack to succeed . . . . . . . . . . . . . . . . . . 10

3.2 Attacking the Boneh-Wu-Zimmerman "Immunized" Variant . . . . . . . . . . . . . . 12

3.3 Attacking the Garg-Gentry-Halevi-Zhandry Countermeasure . . . . . . . . . . . . . . 14

3.4 Attacking GGHRSW Obfuscation for Simple Branching Programs . . . . . . . . . . 16

3.5 Attacking Recent Circuit-Obfuscation Schemes $\ldots \ldots \ldots$. . . . . . . . . . . 19

4 A Weak-DL Attack on Matrix-GGH13 22

4.1 The Updated Weak-DL Attack. . . . . . . . . . . . . . . . . . . . . . 23

\begin{tabular}{|lr}
5 Conclusions & 25
\end{tabular}

\begin{tabular}{lr}
\hline References & 25
\end{tabular}

\begin{tabular}{|lr}
\hline A A Refined Generic Model & 26
\end{tabular}

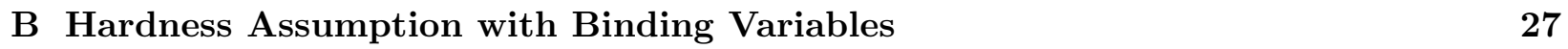

B.1 Attacking Simplified Binding Variables . . . . . . . . . . . . . . . . . . . . 28 


\section{Introduction}

The GGH13 [10] and CLT13 [7] "approximate multilinear maps" candidates suffer from zeroizing attacks, where encodings of zero at levels below the top (zero-test) level can be exploited to recover information that should have been hidden by the encoding scheme. The essence of these attacks is using successful zero tests to obtain equations over the base ring ( $\mathbb{Z}$ or $\mathbb{Z}[X] / F(X)$ ), then solving these equations to get the desired information. First presented in the context of the GGH13 candidate [10, such attacks were recently extended by Cheon et al. [6] also to the CLT13 candidate, where they were shown to be particularly devastating, leading to a total break (when they can be mounted).

As explicitly discussed in [6], however, these attacks seem to depend on the availability of low-level encoding of zeros. This limits the applicability of these attacks, especially since several high-profile applications of multilinear maps (such as for obfuscation [11]) do not reveal such lowlevel zero encodings.

In this work we show that it is possible to "zeroize without low-level zeroes": that is, we extend the attacks from [6] and apply them against both CLT13 encodings and a matrix variant of GGH13 encodings, even in settings where no low-level encodings of zero are available to the adversary. Our extension to the attacks from [6] to avoid low-level zero encodings was also observed independently and concurrently by Boneh, Wu, and Zimmerman [4]. We further systematize the new attacks and show that they can overcome recent proposals to "immunize" against them [12, 4]. In particular, our attacks also deal with cases where more than one monomial is needed to get a zero, and with modifications of the CLT13 and GGH13 schemes that use matrix-based encodings with the encoded values embedded in the eigenvalues of the matrix. Before describing our zeroizing attacks, we discuss the impact and limitations of these attacks.

\section{$1.1 \quad$ Impact of Our Attacks}

Broken Assumptions and Constructions. The most direct consequence of our work is that more hardness assumptions and constructions from the literature are broken. Prior to our work, the attacks of [6] already broke several assumptions and constructions using CLT13 encodings because they provided low-level encodings of zero. Our work extends to new assumptions and constructions, even where no low-level encodings of zero are available. For example, our extensions can be used to break instances of the meta-assumption of Pass et al. [21] (using either GGH13 or CLT13 encodings), even when used without low-level encodings of zero. Furthermore, we show that natural attempts to "immunize" CLT13 or GGH13 encodings by removing low-level encodings of zero [12, 4] fail. In particular, the assumptions used by Gentry et al. [15, 14] are broken, even when "immunized" using the technique from [4]. Perhaps more surprisingly, we also show that simplified variants of certain obfuscation schemes can be broken:

- We show that the GGHRSW branching-program obfuscation procedure from [11], implemented over the CLT13 scheme [7], can be broken when it is applied to branching programs with a very specific "decomposable" structure. See Section 3.4 .

- We also show that the simplified circuit obfuscation scheme of Zimmerman [22, Appendix A] and Applebaum-Brakerski [1] can be broken when applied to very simple circuits (e.g., point functions). 
Generic multilinear model. Zeroizing attacks provide concrete examples of ways in which GGH13 and CLT13 encodings fail to realize the generic multilinear model. That is, they provide natural examples of information that is learnable from certain combinations of CLT13 and GGH13 encodings, but is not learnable in the generic multilinear model. Such attacks of course do not affect the validity of security proofs within the generic multilinear map model, but they illustrate flaws in the model itself when applied to GGH13 and CLT13 encodings.

However, as discussed in more detail below, zeroizing attacks have their limitations, and thus it is important to explore alternative generic models that give the adversary more power, in the hope that they will provide better approximation to the adversarial capabilities against current multilinear-map candidates. Some early attempts along this line can be found in [20, 1]. In the current work we suggest another direction for alternative generic models that would incorporate information learnable by the adversary using zeroizing attacks; see Appendix A.

Subsequent work. Following our work, Badrinarayanan et al. introduced in [2] a generic model that captures all known zeroizing attacks (including ours) by regarding the creation of any encoding of zero as a complete break of the system, and showed how to securely obfuscate evasive functions in this model.

Coron, Lepoint, and Tibouchi recently proposed in [9] a new version of the CLT13 scheme (denoted CLT15), with a modified zero-testing procedure that seems to avoid zeroizing attacks altogether. No polynomial-time attacks are currently known against this version, hence assumptions and constructions that are broken by [6] and our work when instantiated by CLT13 may still hold when using CLT15.

For GGH13, the graded decisional Diffie-Hellman assumption from [10] was broken by Hu and Jia [18], in the same settings for which the initial zeroizing attack [10] applies (i.e., in the presence of low-level encodings of zero).

\subsection{Limitations of Zeroizing attacks}

Potent as they are, zeroizing attacks have their limitations. For example, so far we do not have attacks on any of the $N C^{1}$ obfuscation candidates in the literature. Moreover the "dual-input straddling sets" technique of [3] and its variants that are used in several obfuscation schemes [5, 3, 22 appear to be effective in thwarting these attacks. See more details in Section 2.4.

Successful zero tests are necessary. Our work demonstrate that some attacks are possible even if we only have top-level encoded zeros, but crucially all of these attacks depend on successful zero tests to get equations over the base ring. Some constructions or assumptions may not provide these top-level zeros, and in that case it is plausible that the GGH13 and CLT13 candidates could even provide semantic security [16] of the encoded values. Even more, as far as we know the standard generic multilinear-map model could provide a good approximation of GGH13 and CLT13 in settings where top-level encoding of zeros are not available.

The equations must be simple. In zeroizing attacks, each successful zero-test provides the adversary one equation over the base ring, and the attack relies on the attacker's ability to solve the resulting system of equations. The successful attacks detailed in our paper (as well as those from [10, 6]) arise in situations where the adversary has substantial freedom in creating top-level encodings of zero, and can exploit this freedom to obtain "a simple system of equations" over the base ring that can be solved using linear algebraic techniques.

There are many cases, however, in which the available encodings are constructed such that only 
very particular combinations of them yield a top-level encoding of zero, and those combinations do not seem to yield efficiently solvable system of equations. Two such examples, illustrated in Section 2.4, are obfuscation schemes that rely on Barrington's theorem, and schemes that use the "dual-input straddling sets" technique.

We believe that long-term understanding of the security offered by current multilinear map candidates will require tackling long-standing questions about which kinds of systems of nonlinear equations are feasible to solve efficiently, and which are not.

\section{Background and Overview}

\subsection{A Brief Description of the GGH13 and CLT13 Schemes}

We begin with a brief description of the GGH13 and CLT13 schemes, omitting many details that are irrelevant for the attacks in question. Both these schemes implement graded encoding schemes where "plaintext elements" are encoded in a way that hides their value but allows to add and multiply them, and also allows to test if a degree- $k$ expression in these values is equal to zero (where $k$ is the "multi-linearity parameter").

\subsubsection{The GGH13 scheme}

For GGH13 [10], the plaintext space is a quotient ring $R_{g}=R / g R$ where $R$ is the ring of integers in a number field and $g \in R$ is a "small element" in that ring. The space of encodings is $R_{q}=R / q R$ where $q$ is a "big integer". An instance of the scheme relies on two secret elements, the generator $g$ itself and a uniformly random denominator $z \in R_{q}$. A plaintext element (which is a coset $a=\alpha+g R)$ is encoded "at level one" as $u=[e / z]_{q}$ where $e$ is a "small element" in the coset $a$ (i.e., $e=\alpha+g r$ for some $r \in R$ ). More generally, a level- $i$ encoding of the coset $a$ has the form $u=\left[e / z^{i}\right]_{q}$ for a small $e \in \alpha+g R$.

Addition/subtraction of encodings at the same level is just addition in $R_{q}$, and it results in an encoding of the sum at the same level, so long as the numerators do not wrap around modulo $q$. Similarly multiplication of elements at levels $i, i^{\prime}$ is a multiplication in $R_{q}$, and as long as the numerators do not wrap around modulo $q$ the result is an encoding of the product at level $i+i^{\prime}$.

The scheme also includes a "zero-test parameter" in order to enable testing for zero at level $k$. Noting that a level- $k$ encoding of zero is of the form $u=\left[\mathrm{gr} / z^{k}\right]_{q}$, the zero-test parameter is an element of the form $\mathbf{p}_{\mathrm{zt}}=\left[h z^{k} / g\right]_{q}$ for a "somewhat small element" $h \in R$. This lets us eliminate the $z^{k}$ in the denominator and the $g$ in the numerator by computing $\left[\mathbf{p}_{\mathrm{zt}} \cdot u\right]_{q}=h \cdot r$, which is much smaller than $q$ because both $h, r$ are small. If $u$ is an encoding of a non-zero $\alpha$, however, then multiplying by $\mathbf{p}_{\mathrm{zt}}$ leaves a term of $[h \alpha / g]_{q}$ which is not small. Testing for zero therefore consists of multiplying by the zero-test parameter modulo $q$ and checking if the result is much smaller than $q$.

Matrix-GGH13. An unpublished variant of GGH13 (that was meant to protect against zeroizing attacks) uses matrices of native GGH13 encodings, where the encoded value is an eigenvalue of the matrix and the zero-test parameter includes also the corresponding eigenvector. This is essentially the same as the GGHZ countermeasure construction from [12, Sec. 7] (which is described in Section 3.3), except that it uses GGH13 encodings rather than CLT13 encodings ${ }^{1}$

\footnotetext{
${ }^{1}$ Our attack from Section 3.3 applies for the most part to this GGH13 variant too, except that in this case we only get a weak-DL attack rather than a complete break; see the full version for details.
} 


\subsubsection{The CLT13 Scheme}

The CLT13 scheme [7] is similar to above, but it relies on CRT representation modulo a composite integer $x_{0}=\prod_{j=1}^{n} p_{j}$, where the $p_{j}$ 's are "large primes", all of about the same size. We let $\operatorname{CRT}\left(a_{1}, \ldots, a_{t}\right)$ denote the unique element $a \in \mathbb{Z}_{x_{0}}$ that is congruent to $a_{j}$ modulo $p_{j}$ for all $j$. Also we often use the shorthand CRT $\left(a_{j}\right)_{j}$ to denote the same $2^{2}$

The plaintext space in CLT13 consists of vectors $\mathbf{a} \in \mathbb{Z}_{g_{1}} \times \cdots \times \mathbb{Z}_{g_{n}}$, where all the $g_{j}$ 's are much smaller than their corresponding $p_{j}$ 's. An instance of the scheme relies on the secrets $g_{j}$ and $p_{j}$ (with $x_{0}$ public), and on a secret uniformly random denominator $z \in \mathbb{Z}_{x_{0}}$. Such a vector $\mathbf{a}=\left(\alpha_{1}, \ldots, \alpha_{n}\right)$ is encoded at level one as [CRT $\left.\left(\alpha_{1}+g_{1} r_{1}, \ldots, \alpha_{n}+g_{n} r_{n}\right) / z\right]_{x_{0}}$, where the $r_{j}$ 's are all small. More generally a level- $i$ encoding of this vector is of the form [CRT $\left.\left(\alpha_{j}+g_{j} r_{j}\right)_{j} / z^{i}\right]_{x_{0}}$.

Addition/subtraction of encodings at the same level is just addition in $\mathbb{Z}_{x_{0}}$, and it results in an encoding of the sum at the same level, so long as the numerators in the different CRT components do not wrap around modulo their respective $p_{j}$ 's. Similarly multiplication of elements at levels $i, i^{\prime}$ is a multiplication in $\mathbb{Z}_{x_{0}}$, and as long as the numerators in the different CRT components do not wrap around modulo their respective $p_{j}$ 's, the result is an encoding at level $i+i^{\prime}$ of the entry- wise product of the two vectors.

For zero-testing, let us denote $p_{j}^{*}=x_{0} / p_{j}=\prod_{i \neq j} p_{i}$, and note the following easy corollary of the Chinese Remainder Theorem:

Proposition 2.1. For all $a_{1}, \ldots, a_{n} \in \mathbb{Z}, \mathrm{CRT}\left(p_{j}^{*} a_{j}\right)_{j}=\sum_{j=1}^{n} p_{j}^{*} a_{j}\left(\bmod x_{0}\right)$.

Namely when each CRT component $j$ is divisible by $p_{j}^{*}$, then the CRT composition can be computed just by adding all the CRT components modulo $x_{0}$.

The zero-test parameter in CLT13 is $\mathbf{p}_{\mathrm{zt}}=\left[z^{k} \cdot \mathrm{CRT}\left(p_{j}^{*} h_{j} g_{j}^{-1}\right)_{j}\right]_{x_{0}}$ for small elements $h_{j} \ll p_{j}$, where $g_{j}^{-1}$ is computed modulo $p_{j}$. Multiplying this zero-test parameter by a level- $k$ encoding of zero, that has the form $u=\left[\mathrm{CRT}\left(g_{j} r_{j}\right)_{j} / z^{k}\right]_{x_{0}}$, yields

$$
\left[\mathbf{p}_{\mathrm{zt}} \cdot u\right]_{x_{0}}=\mathrm{CRT}\left(p_{j}^{*} h_{j} r_{j}\right)_{j}=\sum_{j} p_{j}^{*} h_{j} r_{j}
$$

Since $h_{j} r_{j} \ll p_{j}$ for all $j$, then $p_{j}^{*} h_{j} r_{j}=\left(x_{0} / p_{j}\right) h_{j} r_{j} \ll x_{0}$, and also the sum is much smaller than $x_{0}$. Testing for zero therefore consists of multiplying by the zero-test parameter modulo $x_{0}$ and checking if the result is much smaller than $x_{0}$.

\subsubsection{Common Properties}

The GGH13 and CLT13 schemes share a very similar structure; here we summarize the common features that are used in the attacks:

- Each encoding is "associated" with the vector of small integers in the numerator. For GGH13 this is a 1-vector consisting of a single algebraic integer $3^{3}$ and for CLT13 this is a vector of $n$ integers in $\mathbb{Z}$. Below we write informally $u \sim\left(a_{1}, \ldots, a_{n}\right)$ to denote the fact that the encoding $u$ is associated with the vector of $a_{i}$ 's. Roughly speaking, the goal of the attacks is to recover the vector $\left(a_{j}\right)_{j}$ from the encoding $u$. Recovering this vector (even if not in full) is usually considered a break of the scheme.

\footnotetext{
${ }^{2}$ We do not assume that the $a_{j}$ 's are smaller than their corresponding $p_{j}$ 's.

${ }^{3}$ The matrix-GGH13 variant has vectors in the numerator rather than a single algebraic integer.
} 
- An encoding of zero is associated with a vector divisible by the $g_{j}$ 's, namely $u \sim\left(g_{j} r_{j}\right)_{j}$ for some $r_{j}$ 's.

- Addition and multiplication of encodings acts entry-wise on the vector of integers in the numerator. Importantly, the addition and multiplication of these vectors is done over the integers, with no modular reduction. This is because a wrap-around in these operations is an error condition, and so the parameters are always set to ensure that it does not happen.

- If $u \sim\left(g_{j} r_{j}\right)_{j}$ is an encoding of zero at the top level, then applying the zero-test to $u$ returns the integer $w=\sum_{j} r_{j} \rho_{j}$, where the $r_{j}$ 's are the multipliers from the numerator vector and the $\rho_{j}$ 's are system parameters independent of $u$.

In other words, applying the zero-test to an encoding of zero yields the inner-product of the associated vector (sans the $g_{j}$ 's) with a fixed secret vector. (In GGH13 this is the 1-vector $(h)$, in CLT13 the vector is $\left(p_{j}^{*} h_{j}\right)_{j}$.) Importantly, here too the inner product is over the integers, with no modular reduction.

\subsection{Overview of Existing Attacks}

The GGH13 Zeroizing Attack. The following "zeroizing" attack on the GGH13 scheme was described in [10]. It gets as input a level- $t$ encoding of zero $u_{0} \sim(g r)$ and many other level$(k-t)$ encodings $u_{m} \sim\left(a_{m}\right)$. Multiplying $u_{0}$ by any of the $u_{m}$ 's yields a top-level encoding of zero $u_{0} u_{m} \sim\left(g r a_{m}\right)$, and applying the zero-test yields the algebraic integer $w_{m}=h r a_{m}$. Note that this almost recovers the numerators $a_{m}$ 's; indeed we have them up to the common factor $h^{\prime}=h r$.

If we also knew the ideal $I_{g}=g R$ that defines the plaintext space, then being able to recover the numerator up to a constant is enough to break many hardness assumptions. For example, given an encoded matrix we could compute its determinant $\left(\bmod I_{g}\right)$ up to a constant, which would tell us whether or not the encoded matrix has full rank.

Even when $I_{g}$ is not explicitly given, Garg et al. described in [10] how it can be recovered in certain cases using GCD computations. Roughly, we can use GCD to identify and remove the common factor $h^{\prime}$, thereby getting the $a_{m}$ 's themselves, except that these are all algebraic integers so we only have GCD in terms of their ideals. Recovering the ideal $I_{a}=a R$ is not always useful, e.g., if $I_{a}$ and $I_{g}$ are co-prime then knowing $I_{a}$ does not tell us anything about our plaintext coset $a+I_{g}$. However if some of the $u_{i}$ 's are themselves encoding of zero, namely $a_{i}=g r_{i}$, then given enough ideals $I_{a_{i}}=g r_{i} R$ we could again use GCD calculations to recover the ideal $I_{g}$ itself, and then use that knowledge to attack the non-zero encodings among the $u_{i}$ 's. This attack was called in [10] a "weak discrete-log attack". Recently, this attack was used by Hu and Jia [18] as a component in a new attack that breaks the key-exchange protocol from [10].

We note that the GGH13 zeroizing attack does not work against CLT13 encodings, since rather than a simple product we now have an inner product $w_{m}=\sum_{j} a_{m, j} \rho_{j}$, and we cannot use this to compute GCDs. (For the same reason, this attack does not work against the matrix-GGH13 variant.)

The CHLRS Zeroizing Attack. Cheon, Han, Lee, Ryu and Stehlé recently described in [6] a major upgrade of the GGH13 zeroizing attack, which can be used to completely break CLT13based schemes in some cases, recovering the factorization of $x_{0}$ and all secret information. To mount the CHLRS zeroizing attack we need three sets of encoded inputs, which we denote by 
$\mathcal{A}=\left\{A_{i}: i=1, \ldots, n\right\}, \mathcal{B}=\left\{B_{0}, B_{1}\right\}$, and $\mathcal{C}=\left\{C_{j}: j=1, \ldots, n\right\}$ (with $n$ the dimension of the numerator vectors). The $A$ 's are all random encoding of zeros, the $B$ 's are the target of the attack, and the $C$ 's are just helper encodings of random vectors. The levels of these encodings are such that multiplying $A_{i} \cdot B_{\sigma} \cdot C_{j}$ yields a top-level encoding of zero for any $i, \sigma, j$. Below we denote the numerator vectors associated with these encodings by

$$
A_{i} \sim\left(g_{1} r_{i, 1}, \ldots, g_{n} r_{i, n}\right), B_{\sigma} \sim\left(b_{\sigma, 1}, \ldots, b_{\sigma, n}\right), \text { and } C_{j} \sim\left(c_{j, 1}, \ldots, c_{j, n}\right) .
$$

Multiplying $A_{i} \cdot B_{\sigma} \cdot C_{j}$ yields a top-level encoding of zero, associated with the vector $A_{i} \cdot B_{\sigma} \cdot C_{j} \sim$ $\left(g_{1} r_{i, 1} b_{\sigma, 1} c_{j, 1}, \ldots, g_{n} r_{i, n} b_{\sigma, n} c_{j, n}\right)$. Applying the zero-test we get a four-wise inner product, yielding the integer $w_{\sigma}[i, j]=\sum_{k=1}^{n} \rho_{k} r_{i, k} b_{\sigma, k} c_{j, k}$. We can write this four-wise inner product in matrix form as

$$
w_{\sigma}[i, j]=\left(\begin{array}{lll}
r_{i, 1} & \ldots & r_{i, n}
\end{array}\right) \times\left(\begin{array}{ccc}
\rho_{1} b_{\sigma, 1} & & \\
& \ddots & \\
& & \rho_{n} b_{\sigma, n}
\end{array}\right) \times\left[\begin{array}{c}
c_{j, 1} \\
\vdots \\
c_{j, n}
\end{array}\right],
$$

and denote the vector on the left by $\mathbf{a}_{i}$, the matrix in the middle by $B_{\sigma}^{\prime}$, and the vector on the right by $\mathbf{c}_{j}$. For a fixed $\sigma$, let $i, j$ range over $1, \ldots, n$. This yields an $n \times n$ matrix of integers $W_{\sigma}=\left[w_{\sigma}[i, j]\right]_{i, j}=A^{\prime} \times B_{\sigma}^{\prime} \times C^{\prime}$, where $A^{\prime}$ has the $\mathbf{a}_{i}$ 's for rows and $C^{\prime}$ has the $\mathbf{c}_{j}$ 's for columns. Since the $r_{i, k}$ 's, $b_{\sigma, k}$ 's, $c_{j, k}$ 's and $\rho_{k}$ 's are all random (small) quantities, then with high probability the matrices are all invertible (over the rationals). Having computed the matrices $W_{\sigma}$, the attacker now sets

$$
W=W_{0} \times W_{1}^{-1}=\left(A^{\prime} B_{0}^{\prime} C^{\prime}\right) \times\left(A^{\prime} B_{1}^{\prime}, C^{\prime}\right)^{-1}=A^{\prime} \times\left(B_{0}^{\prime} \times B_{1}^{\prime-1}\right) \times A^{\prime-1} .
$$

Observe now that $B^{*}=B_{0}^{\prime} \times B_{1}^{\prime-1}$ is a diagonal matrix with $b_{0, j} / b_{1, j}$ on the diagonal, and thus the eigenvalues of $B^{*}$ are all the ratios $b_{0, j} / b_{1, j}$. And since $W$ and $B^{*}$ are similar matrices, then also the eigenvalues of $W$ are the $b_{0, j} / b_{1, j}$ 's. Hence once it computes $W$, the attacker can find its eigenvalues (over the rationals) and obtain all the ratios $b_{0, j} / b_{1, j}$.

These ratios may be enough by themselves to break some hardness assumptions, but for CLT13 it is possible to use them to factor $x_{0}$, thereby getting a complete break. Specifically, since each ratio is rational it can be written as $u / v=b_{0, j} / b_{1, j}$ with $u, v$ co-prime integers. Recalling now that $B_{0}, B_{1}$ are two encodings at the same level (say, level $t$ ) with numerator vectors $\left(b_{0,1}, \ldots, b_{0, n}\right.$ ) and $\left(b_{1,1}, \ldots, b_{1, n}\right)$, respectively, we get that

$$
u B_{1}-v B_{0}=\left[\mathrm{CRT}\left(u b_{1,1}-v b_{0,1}, \ldots, u b_{1, n}-v b_{0, n}\right) / z^{t}\right]_{x_{0}} .
$$

This means that the $j$ 'th CRT component is $u b_{1, j}-v b_{0, j}=0$, and with high probability the others are not, so we get $G C D\left(x_{0}, u B_{1}-v B_{0}\right)=p_{j}$.

\subsection{Extending the CHLRS Attack}

In the current work we describe several extensions to attacks of Cheon et al. from [6]; below we describe these extensions briefly.

\subsubsection{GGH13 vs. CLT13}

We can also apply these zeroizing attacks to a matrix variant of GGH13, not just to CLT13 encodings, resulting in a "weak discrete-log" attack. This is described in Section 4. 


\subsubsection{Orthogonal encodings}

We also note that these attacks do not actually require low-level encoding of zeros. Indeed all we need is that for every $i, \sigma, j$, the product $A_{i} B_{\sigma} C_{j}$ is a top-level encoding of zero, so we could have the $A$ 's with zeros in a few CRT components, the $B$ 's with zeros in some other components, and the $C$ 's with zeros in all the CRT components not covered by the $A$ 's and $B$ 's. This observation was also made concurrently by Boneh et al. [4].

\subsubsection{More than one monomial}

The attack also extends to a setting where more than a single monomial is needed to get a zero. For example, consider the case where we have not three but six sets of encodings. Similar to before we have $\mathcal{A}=\left\{A_{i}: i=1, \ldots, 2 n\right\}, \mathcal{B}=\left\{B_{0}, B_{1}\right\}$, and $\mathcal{C}=\left\{C_{j}: j=1, \ldots, 2 n\right\}$, but now we also have $\tilde{\mathcal{A}}=\left\{\tilde{A}_{i}: i=1, \ldots, 2 n\right\}, \tilde{\mathcal{B}}=\left\{\tilde{B}_{0}, \tilde{B}_{1}\right\}$, and $\tilde{\mathcal{C}}=\left\{\tilde{C}_{j}: j=1, \ldots, 2 n\right\}$. (Note that the indices $i, j$ now range over $[1,2 n]$, not $[1, n])$. The new attack requires that $A_{i} B_{\sigma} C_{j}+\tilde{A}_{i} \tilde{B}_{\sigma} \tilde{C}_{j}$ is a top-level encoding of zero for every $i, \sigma, j$. We denote the numerator vectors associated with these encodings by

$$
\begin{aligned}
& A_{i} \sim\left(a_{i, 1}, \ldots, a_{i, n}\right), \quad B_{\sigma} \sim\left(b_{\sigma, 1}, \ldots, b_{\sigma, n}\right), \quad C_{j} \sim\left(c_{j, 1}, \ldots, c_{j, n}\right), \\
& \tilde{A}_{i} \sim\left(\tilde{a}_{i, 1}, \ldots, \tilde{a}_{i, n}\right), \quad \tilde{B}_{\sigma} \sim\left(\tilde{b}_{\sigma, 1}, \ldots, \tilde{b}_{\sigma, n}\right), \quad \tilde{C}_{j} \sim\left(\tilde{c}_{j, 1}, \ldots, \tilde{c}_{j, n}\right) .
\end{aligned}
$$

We can think of the pairs $\left(A_{i}, \tilde{A}_{i}\right),\left(B_{\sigma}, \tilde{B}_{\sigma}\right),\left(C_{j}, \tilde{C}_{j}\right)$ as encodings that are associated with numerator vectors of twice the dimension, and the CHLRS attack can be applied to these new "double encodings". The only difference (other than the larger dimension) is that we can no longer associate the division-by- $g_{i}$ with any single vector. Instead, applying the zero-test to $A_{i} B_{\sigma} C_{j}+$ $\tilde{A}_{i} \tilde{B}_{\sigma} \tilde{C}_{j}$ yields a four-wise inner product divided by the $g_{i}$ 's, which we can write in matrix form:

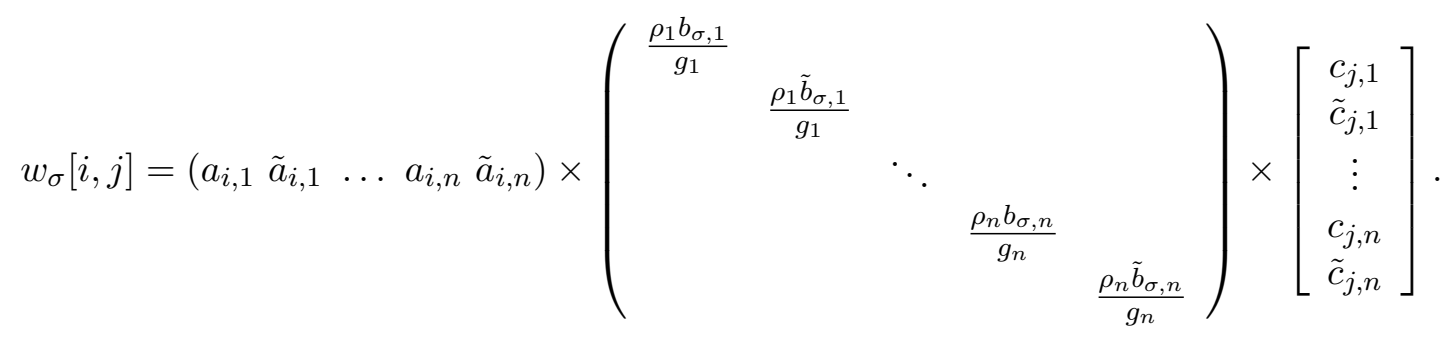

Importantly, even though we have division by $g_{i}$ 's, this equation holds over the rationals, without modular reduction. The attack itself proceeds just as before, and the $g_{i}^{-1}$ factors conveniently fall off when we compute $B_{0}^{\prime} \times B_{1}^{\prime-1}$. This extension can be used to break the "immunized" CLT13 variant from [4].

\subsubsection{Using Cayley-Hamilton}

In response to the CHLRS attacks, Garg et al. described in [12, Sec. 7] a variant of the CLT13 encoding that uses matrices for encoding, rather than single $\mathbb{Z}_{x_{0}}$ elements (see description in Section 3.3 below).

The attacks above apply also to this variant for the most part, but the resulting matrices $B_{0}^{\prime}, B_{1}^{\prime}$ are no longer diagonal. Instead they are block-diagonal with the block dimension corresponding to the dimension of the encoding matrices, and different blocks corresponding to different CRT 
components (i.e. $B_{\sigma} \bmod p_{j}$ ). The eigenvalues of $B_{0}^{\prime} \times B_{1}^{\prime-1}$ in this case need not be rational numbers anymore, they can be arbitrary complex numbers, and so the final step in the CHLRS attack cannot be applied.

However the characteristic polynomial of $B^{*}=B_{0}^{\prime} \times B_{1}^{\prime-1}$ is still the product of the characteristic polynomials of the blocks. We can factor the characteristic polynomial of $B^{*}$ to find the block characteristic polynomials, and then apply these block polynomials to the matrix $M=B_{1} \times B_{0}^{-1}$. Applying a block polynomial to $M$ zeros out the corresponding CRT component (by the CayleyHamilton theorem), but not the others (whp), and we can then compute the GCD of $x_{0}$ and any matrix element to recover the prime corresponding to the zeroed CRT component. Note this assumes that the block polynomials are irreducible over $\mathbb{Q}$ (which indeed holds for [12, Sec. 7]), so that they can be efficiently found by factoring $B^{*}$ 's characteristic polynomial.

The actual procedure that we use differs slightly, in order to handle an unpublished generalization of [12, Sec. 7] in which the encoding matrices themselves are constructed to be block-diagonal, say with block dimension $d$. With this change $B^{*}$ is still block-diagonal, but the block dimension is now larger by a factor of $d$, and each polynomial that we want to apply to $M$ is the product of $d$ factors of $B^{*}$ 's characteristic polynomial. We do not know of a way to efficiently partition these factors into the correct sets of size $d$. Instead, we remove one irreducible factor from $B^{*}$ 's characteristic polynomial, and apply the resulting polynomial to $M$. This has the effect of zeroing out all CRT components except the one corresponding to the removed factor, so computing the GCD with $x_{0}$ recovers the product of all but one of the primes, and dividing $x_{0}$ by this recovers an individual prime. Cycling over all irreducible factors, we recover all of the primes.

\subsection{Attack Limitations}

As sketched in the introduction, zeroizing attacks have their limitations, in that they require zeros and moreover need the equations that yield these zeros to be "simple." Two scenarios that seem outside the scope of these attacks due to "non-simple" equations are discussed next.

Obfuscation using Barrington's theorem. Consider the obfuscation schemes in the literature that obfuscate matrix-based branching programs (BP) resulting from Barrington's theorem [11, 5, 3, 21. These schemes are designed so that the only way to get a top-level zero encoding is using the prescribed routines for evaluating the obfuscated circuit on various inputs, so we only need to examine the type of expressions that arise from such evaluation.

Recall that a matrix-based BP has a sequence of steps, each specified by two matrices and controlled by an input bit. On a given input, we choose one of the two matrices in each step (based on the corresponding input bit), then multiply all of the selected matrices in order to get the result. In the BPs that are generated by Barrington's theorem, each input bit controls several steps that are spaced far apart, and so changing the value of that bit changes the selection of all these matrices. This makes it hard to apply our attacks in this setting, since these attacks require a multilinear setting where we can get many different zeros by changing just a single variable in every monomial. Therefore, even though we do get equations over the base ring from top-level zeros in this scheme, these equations appear to be correlated in a highly non-linear manner, foiling our attempts to glean useful information from them. (Rather than the individual matrices, one can consider for each bit position $j$ two tensor products of matrices corresponding to this bit position, one tensor of the matrices for value 0 and the other for the value 1 . Then we can get a multilinear equations in these tensor vectors, but we do not see how to use them to mount a successful attack.) 
We contrast this situation with the attack that we describe in Section 3.4, that breaks obfuscation of very simple branching programs which are "separable" in the sense that different subsets of the input bits control different consecutive intervals of steps, thus giving us the simple system of equations that we need.

Binding variables. The CHLRS attacks and our extensions rely on the ability to partition the variables into groups $(\mathcal{A}, \mathcal{B}, \mathcal{C}$ above), so that we can independently choose variables from the different groups and every such choice yields a top-level zero. Several schemes in the literature use explicit binding variables to make it hard to partition the encodings into independent sets. For example, the obfuscation schemes of Barak et al. [3] and Zimmerman [22] use "dual-input straddling sets" to create a "high connectivity" interlocking set of encodings.

These schemes contain, for each pair $i, j$ of input bits, four encoded variables $U_{i, j, 0,0}, U_{i, j, 0,1}, U_{i, j, 1,0}$, and $U_{i, j, 1,1}$, such that obtaining a top-level encoding of zero requires multiplying $U_{i, j, *, *}$ 's that are consistent with some $n$-bit input $x$ (i.e., it requires computing some expression $\cdot \prod_{i, j} U_{i, j, x_{i}, x_{j}}$ ). This structure seems to foil attempts of separating the variables into independent sets, since changing any input bit creates a cascading effect. To illustrate the difficulty of applying the attack in this setting, we describe in Appendix B, a relatively simple source-group hardness assumption involving such binding variables, which we do not know how to break even though we are given many lowlevel CLT13 encodings of zero. We mention that in some simple cases one can use GCDs to remove the binding variables and mount the same attack; this is described in Appendix B.1. However we see no way of extending that attack to the binding variables as they are used in the literature.

\section{A Unified Attack against CLT13-Based Schemes}

Below we present a general attack on CLT13-based schemes that combines all the ideas from Section 2.3, and show how this attack can be used against:

- The CLT13 scheme with orthogonal encodings (details omitted), and the proposed modifications by Boneh et al. [4] and Garg et al. [12, Sec. 7] (that were suggested in response to the CHLRS attacks);

- Obfuscations of branching programs with specific structure using the iO procedure of Garg et al. [11]; and

- Obfuscations of simple circuits (such as point functions) when the "simplified iO variant" of Zimmerman [22, App. A] and Applebaum and Brakerski [1].

Central to our general attack is the notion of a "good attack set," which roughly plays the role of the sets $\mathcal{A}, \mathcal{B}, \mathcal{C}$ from Section 2 (together with the zero-test parameter). To define this notion formally, fix an instance of CLT13 with $n$ secret primes $p_{1}, \ldots, p_{n}$ and modulus $x_{0}:=\prod_{i} p_{i}$. An attack set (of dimension $d$ ) consists of three sets of matrices $\mathcal{A}, \mathcal{B}, \mathcal{C} \subset \mathbb{Z}_{x_{0}}^{d \times d}$, of sizes $|\mathcal{A}|=|\mathcal{C}|=n d$ and $|\mathcal{B}|=2$, and two vectors $s \in \mathbb{Z}_{x_{0}}^{1 \times d}$ and $t \in \mathbb{Z}_{x_{0}}^{d \times 1}$. These sets are constructed from the available public parameters and encodings of a given scheme, in such a way that for every choice of $\left(A_{i}, B_{\sigma}, C_{j}\right) \in \mathcal{A} \times \mathcal{B} \times \mathcal{C}$, the value

$$
W_{\sigma}[i, j]:=s \times A_{i} \times B_{\sigma} \times C_{j} \times t \in \mathbb{Z}_{x_{0}}
$$


Input: $\mathcal{A}=\left\{A_{i}\right\}_{i}, \mathcal{B}=\left\{B_{\sigma}\right\}_{\sigma}, \mathcal{C}=\left\{C_{j}\right\}_{j}, s, t$

1. Compute $(n d) \times(n d)$ matrices $W_{0}, W_{1}$ as $W_{\sigma}[i, j]:=\left[s \times A_{i} \times B_{\sigma} \times C_{j} \times t\right]_{x_{0}}$.

2. Compute $W:=W_{0} \times W_{1}^{-1}$ over $\mathbb{Q}$, and $M:=B_{0} \times B_{1}^{-1}\left(\bmod x_{0}\right)$.

3. Compute $W$ 's characteristic polynomial $f:=\operatorname{charPoly}(W)$ over $\mathbb{Q}$, and factor it into monic irreducible factors over $\mathbb{Q}$ as $f=f_{1} f_{2} \cdots f_{m}$.

4. For all $k \in\{1, \ldots, m\}$ define $F_{k}:=f / f_{k}=\prod_{i \neq k} f_{i} \in \mathbb{Q}[X]$, let $d_{k}$ be the common denominator of the coefficients of $F_{k}$, and set $G_{k}:=F_{k} \cdot d_{k}$.

5. Evaluate the $G_{k}$ 's at the matrix $M \bmod x_{0}, M_{k}:=\left[G_{k}(M)\right]_{x_{0}} \forall k \leqslant m$.

6. Compute $S:=\left\{G C D\left(M_{k}[i, j], x_{0}\right) \mid i, j \in[n d] ; k \in[m]\right\}$, and return $\left\{x_{0} / q \mid q \in S\right\}$.

Figure 1: Our general attack on CLT13-based schemes

is a zero-tested top-level encoding of 0. (The CHLRS attack can be thought as a special case where all the "matrices" are of dimension $d=1$, and we have $s=1$ and $t=\mathbf{p}_{\mathrm{zt}}$.) Given such an attack set, the attack proceeds as in Figure 1, where we denote by $[z]_{p}$ the reduction of $z$ modulo $p$ into the interval $[-p / 2, p / 2)$, and this notation extends entry-wise to vectors and matrices.

\subsection{Sufficient conditions for the attack to succeed}

Next we state and prove sufficient conditions on the attack set that ensures that the attack in Figure 1 succeeds. Specifically, we would like to show that each $M_{k}$ in step 5 must be zero modulo all the primes except one, and hence any non-zero entry in it yields a nontrivial factor of $x_{0}$ (i.e. the product of those primes).

Referring to the intuition from Section 2.3.4, the matrix $W=A \times B^{*} \times A^{-1}$ is similar to a block-diagonal matrix $B^{*}$ that has one block for each CRT component. Specifically, the $j$ th block of $B^{*}$ is $B_{j}^{*}=\left[B_{0}\right]_{p_{j}} \times\left(\left[B_{1}\right]_{p_{j}}\right)^{-1}$ (inverse over $\left.\mathbb{Q}\right)$. The characteristic polynomial of $W$ is then the product of the characteristic polynomials of all the blocks. For simplicity, assume the block polynomials are the irreducible factors $f_{i}$ from Figure 1. Then each $F_{k}$ is thus the product of all block polynomials except the $k$ th, and by the Cayley-Hamilton theorem we have that $F_{k}\left(B_{j}^{*}\right)=0$ (and therefore also $G_{k}\left(B_{j}^{*}\right)=0$ ) for all blocks $j \neq k$. But $G_{k}\left(B_{j}^{*}\right)=0$ over $\mathbb{Q}$ implies that also $G_{k}\left(B_{0} \times B_{1}^{-1}\right)=0\left(\bmod p_{j}\right)$, so $G_{k}(M)$ is zero modulo all primes $j \neq k$. The only thing left to ensure is that for the last prime $p_{k}$ we get $G_{k}(M) \neq 0\left(\bmod p_{k}\right)$, which is the essence of our sufficient condition. The actual condition in Definition 1 below is slightly more complex, to account for the case when the block polynomials are reducible over $\mathbb{Q}$.

Definition 1. Fix an attack set $(\mathcal{A}, \mathcal{B}, \mathcal{C}, s, t)$. Let $B_{0}, B_{1}, M, W$ be the matrices from Figure 1 . and let $g_{j}:=$ charPoly $\left(\left[B_{0}\right]_{p_{j}} \times\left[B_{1}\right]_{p_{j}}^{-1}\right)$ over $\mathbb{Q}$. We say that $(\mathcal{A}, \mathcal{B}, \mathcal{C}, s, t)$ is good if:

1. $f:=\operatorname{charPoly}(W)=\prod_{j \leqslant n} g_{j}$;

2. $B_{1}$ is non-singular modulo $x_{0}$;

3. The common denominators $d_{k}$ from step 4 are all co-prime with $x_{0}$; 
4. For any $j \leqslant n$ and any divisor $f_{k}$ of $g_{j}$ of degree $\geqslant 1$ (possibly $f_{k}=g_{j}$ ), denoting $G_{k}=d_{k} \cdot f / f_{k}$ as in step 4 , we have $G_{k}(M) \neq 0\left(\bmod p_{j}\right)$.

Theorem 1. For any good attack set $(\mathcal{A}, \mathcal{B}, \mathcal{C}, s, t)$, the algorithm in Figure 1 recovers the secret primes $p_{1}, \ldots, p_{n}$.

To prove Theorem 1 we use the following lemma:

Lemma 3.1. Let $p>1$ and $u_{1}, \ldots, u_{t}, v_{1}, \ldots, v_{t}$ be integers, s.t. the $v_{i}$ 's are invertible mod $p$, and denote $w_{i}=\left[u_{i} \cdot v_{i}^{-1}\right]_{p}$. If $g$ is a multivariate integer polynomial such that $g\left(\frac{u_{1}}{v_{1}}, \ldots, \frac{u_{t}}{v_{t}}\right)=0$ over $\mathbb{Q}$, then $g\left(w_{1}, \ldots, w_{t}\right)=0(\bmod p)$.

Proof. It is enough to prove it for a linear $g$, since we can replace any non-linear term $\prod_{i \in I}\left(\frac{u_{i}}{v_{i}}\right)^{e_{i}}$ (for some $I \subset[t]$ and $e_{i}$ 's) by new variables $u^{\prime}=\prod_{i \in I} u_{i}^{e_{i}}, v^{\prime}=\prod_{i \in I} v_{i}^{e_{i}}$, and $w^{\prime}=\left[\prod_{i \in I} w_{i}^{e_{i}}\right]_{p}=$ $\left[u^{\prime} \cdot v^{\prime-1}\right]_{p}$, and then prove the same statement on the resulting new polynomial.

Now denote $V=\prod_{i} v_{i}$ and for each $i$ denote $v_{i}^{*}=V / v_{i}=\prod_{j \neq i} v_{j}$. For a linear $g$ we can write $\sum_{i} g_{i} \cdot \frac{u_{i}}{v_{i}}=0$ over $\mathbb{Q}$, so also $\sum g_{i} u_{i} v_{i}^{*}=V \cdot \sum_{i} g_{i} \cdot \frac{u_{i}}{v_{i}}=0$, and in particular $\sum g_{i} u_{i} v_{i}^{*}=0(\bmod p)$. Finally, since $V$ is invertible modulo $p$ we get

$$
\sum_{i} g_{i} w_{i}=\sum_{i} g_{i} u_{i} v_{i}^{-1}=V^{-1} \cdot \sum_{i} g_{i} u_{i} v_{i}^{*}=0 \quad(\bmod p) .
$$

Proof of Theorem 11. For all $i$ denote $B_{i}^{*}=\left[B_{0}\right]_{p_{i}} \times\left[B_{1}\right]_{p_{i}}^{-1}$ over $\mathbb{Q}$ and $\hat{B}_{i}=\left[B_{0}\right]_{p_{i}} \times\left[B_{1}\right]_{p_{i}}^{-1}$ over $\mathbb{Z}_{p_{i}}$. Let $t_{i}:=\operatorname{det}\left(\left[B_{1}\right]_{p_{i}}\right.$ ) (over $\mathbb{Q}$ ), and since $B_{1}$ is non-singular modulo $x_{0}$ then in particular $t_{i} \neq 0$ $\left(\bmod p_{i}\right)$. We can therefore write $B_{i}^{*}=\tilde{B}_{i} / t_{i}$ for an integer matrix $\tilde{B}_{i}$, and clearly we also have $\hat{B}_{i}=\tilde{B}_{i} \cdot t^{-1}\left(\bmod p_{i}\right)$.

Denote the characteristic polynomial of $B_{i}^{*}$ over $\mathbb{Q}$ by $g_{i}:=$ charPoly $\left(B_{i}^{*}\right)$. By the first condition in Definition 1 we have $f:=$ charPoly $(W)=\prod_{j \leqslant n} g_{j}$. Note, however, that the $g_{j}$ 's are not necessarily irreducible, so there isn't necessarily a 1-1 correspondence between the $g_{j}$ 's and the irreducible factors $f_{k}$ of $f$.

Fix an index $j \leqslant n$ and we show that for some $k$ it holds that $G_{k}(M) \neq 0\left(\bmod p_{j}\right)$ but $G_{k}(M)=0\left(\bmod p_{i}\right)$ for all $i \neq j$. Clearly this $g_{j}$ is divisible by at least one $f_{k}$ (which has degree $\geqslant 1)$, so the last condition of Definition 1 implies that $G_{k}(M)=d_{k} \cdot F_{k}(M) \neq 0\left(\bmod p_{j}\right)$. It remains to show that for all the other primes $p_{i}, i \neq j$, we have $G_{k}(M)=0\left(\bmod p_{i}\right)$.

Clearly $F_{k}$ is divisible by $g_{i}$ for every $i \neq j$, so the Cayley-Hamilton theorem implies that $F_{k}\left(B_{i}^{*}\right)=0$ (over $\mathbb{Q}$ ) for all $i \neq j$, and therefore also $G_{k}\left(B_{i}^{*}\right)=0$. Viewing $G_{k}\left(B_{i}^{*}\right)$ as a collection of multivariate polynomials over the elements of $B_{i}^{*}$, and using the facts that $B_{i}^{*}=\tilde{B}_{i} / t_{i}$ and $\hat{B}_{i}=\tilde{B}_{i} \cdot t^{-1}\left(\bmod p_{i}\right)$, we can apply Lemma 3.1 to conclude that also $G_{k}\left(\hat{B}_{i}\right)=0\left(\bmod p_{i}\right)$. And since $M=\hat{B}_{i}\left(\bmod p_{i}\right)$ then also $G_{k}(M)=0\left(\bmod p_{i}\right)$, as needed.

We have shown that $M_{k}:=G_{k}(M)$ satisfies $M_{k} \neq 0\left(\bmod p_{j}\right)$ but $M_{k}=0\left(\bmod p_{i}\right)$ for all $i \neq j$, so there exists an entry $z=M_{k}[a, b]$ such that $z \neq 0\left(\bmod p_{j}\right)$ but $z=0\left(\bmod p_{i}\right)$ for all $i \neq j$. Thus $G C D\left(z, x_{0}\right)=\prod_{i \neq j} p_{i}$, and $x_{0} / G C D\left(z, x_{0}\right)=p_{j}$.

Below we construct good attack sets for some schemes in the literature. We will repeatedly use the fact that for a CLT13 encoding $u$ associated with numerator vector $u \sim\left(r_{i} g_{i}+m_{i}\right)_{i}$, the randomization vector $\left(r_{i}\right)_{i \in[n]}$ is nearly uniform for each encoding. Specifically we have the following, which is proved in [4, Lemma 5.7]. 
Lemma $3.2\left([4)\right.$. There exists a prime $q=2^{\Omega(n)}$ which is determined by the CLT13 system parameters such that, for each encoding, the distribution on $\left(r_{i} \bmod q\right)_{i \in[n]}$ is $\operatorname{neg}(n)$-close to the uniform distribution on $\mathbb{Z}_{q}^{n}$.

\subsection{Attacking the Boneh-Wu-Zimmerman "Immunized" Variant}

Boneh, Wu and Zimmerman [4] proposed an "immunization" of the CLT13 scheme, which more generally applies to any composite-order asymmetric graded encoding scheme. At a high level, the idea is to add two additional slots to the underlying scheme, and use these to maintain functionality while preventing the possibility of having two encodings that multiply to 0 at the top level.

For purposes of illustration, a BWZ-encoding of plaintext $m$ is given by two CLT13-encodings $\left[a, a^{\prime}\right]: a$ encodes $\left(m, \alpha, \beta_{1}\right)$ at level $z_{1}$ and $a^{\prime}$ encodes $\left(\beta_{2}, \alpha, \beta_{3}\right)$ at level $z_{2}$, where $\alpha$ and the $\beta_{i}$ are independent and uniform in the appropriate range. Two additional CLT13-encodings are given out for zero testing: $t_{L}$ encodes $(1,1,0)$ at level $z_{2}$, and $t_{R}$ encodes $(0,1,0)$ at level $z_{1}$. The top encoding level is $z_{1} z_{2}$. One can add and multiply BWZ-encodings component-wise, and given a BWZ-encoding $\left[a, a^{\prime}\right]$ one can check if it is zero by computing the top-level CLT13 encoding $t_{L} \cdot a-t_{R} \cdot a^{\prime}$. With high probability this will be a CLT13-encoding of $(0,0,0)$ iff $\left[a, a^{\prime}\right]$ was a BWZ-encoding of 0. (Note that [4] can handle a general asymmetric level structure; we chose a simple one here for clarity.)

The motivation for this scheme is that it will never give out two CLT13-encodings $a$ and $b$ such that $a b$ is a top-level CLT13-encoding of 0 . However, $\left(t_{L} \cdot a-t_{R} \cdot a^{\prime}\right) \cdot \mathbf{p}_{\mathrm{zt}}$ is still a $2(n+2)$ linear form in the CRT components of $a$ and $a^{\prime}$, and we can extend the Cheon et al. attack to this setting.

Attack Sets. We construct the three sets of matrices $\mathcal{A}, \mathcal{B}, \mathcal{C}$ for the attack on the scheme as follows. We start with three sets of BWZ-encodings, which can be obtained from the ZMM.Encode function of [4, Construction 3.1]:

- $\left\{\left[\hat{a}_{i}, \hat{a}_{i}^{\prime}\right]: i=1, \ldots, 2 n^{\prime}\right\}: \hat{a}_{i}$ at level $z_{a}$ and $\hat{a}_{i}^{\prime}$ at level $z_{a}^{\prime}$.

- $\left\{\left[\hat{b}_{\sigma}, \hat{b}_{\sigma}^{\prime}\right],: \sigma=0,1\right\}: \hat{b}_{\sigma}$ at level $z_{b}$ and $\hat{b}_{\sigma}^{\prime}$ at level $z_{b}^{\prime}$.

- $\left\{\left[\hat{c}_{j}, \hat{c}_{j}^{\prime}\right]: j=1, \ldots, 2 n^{\prime}\right\}: \hat{c}_{j}$ at level $z_{c}$ and $\hat{c}_{j}^{\prime}$ at level $z_{c}^{\prime}$.

Here $n^{\prime}:=n+2$ is the number of slots in each component of a BWZ-encoding (i.e. $n$ is the number of slots in the "un-immunized" scheme.) The scheme also gives out two other encodings: $\hat{t}_{L}$ at level $z_{a}^{\prime} z_{b}^{\prime} z_{c}^{\prime} z_{T}$ and $\hat{t}_{R}$ at level $z_{a} z_{b} z_{c} z_{T}$, where the top CLT13 encoding level is $z:=z_{a} z_{a}^{\prime} z_{b} z_{b}^{\prime} z_{c} z_{c}^{\prime} z_{T}$. These encodings have the property that for all $i, \sigma, j:\left(\hat{a}_{i} \hat{b}_{\sigma} \hat{c}_{j}, \hat{a}_{i}^{\prime} \hat{b}_{\sigma}^{\prime} \hat{c}_{j}^{\prime}\right)$ is a top-level BWZ encoding of 0, i.e. $\hat{t}_{L} \hat{a}_{i} \hat{b}_{\sigma} \hat{c}_{j}-\hat{t}_{R} \hat{a}_{i}^{\prime} \hat{b}_{\sigma}^{\prime} \hat{c}_{j}^{\prime}$ is a top-level CLT13-encoding of 0 .

Then we set

$$
\mathcal{A}=\left\{A_{i}=\left[\begin{array}{cc}
\hat{a}_{i} & 0 \\
0 & \hat{a}_{i}^{\prime}
\end{array}\right]\right\} \quad \mathcal{B}=\left\{B_{\sigma}=\left[\begin{array}{cc}
\hat{b}_{\sigma} & 0 \\
0 & \hat{b}_{\sigma}^{\prime}
\end{array}\right]\right\} \quad \mathcal{C}=\left\{C_{j}=\left[\begin{array}{cc}
\hat{c}_{j} & 0 \\
0 & \hat{c}_{j}^{\prime}
\end{array}\right]\right\} .
$$

Finally we set the vectors $s=\left[\hat{t}_{L}, 1\right]$ and $t=\mathbf{p}_{\mathrm{zt}} \cdot\left[1,-\hat{t}_{R}\right]^{T}$, where

$$
\mathbf{p}_{\mathrm{zt}}=\left[z \cdot \operatorname{CRT}\left(p_{k}^{*} h_{k} g_{k}^{-1} \bmod p_{k}\right)_{k}\right]_{x_{0}}
$$

is the zero-testing parameter of the underlying CLT13 scheme (as explained in Section 2.1.2). 
Set Properties. Next we show that $(\mathcal{A}, \mathcal{B}, \mathcal{C}, s, t)$ form a good attack set according to Definition 1 . First, we observe that

$$
W_{\sigma}[i, j]=s \times A_{i} \times B_{\sigma} \times C_{j} \times t=\left(\left[\hat{a}_{i} \hat{b}_{\sigma} \hat{c}_{j}, \hat{a}_{i}^{\prime} \hat{b}_{\sigma}^{\prime} \hat{c}_{j}^{\prime}\right] \cdot\left[\hat{t}_{L},-\hat{t}_{R}\right]\right) \mathbf{p}_{\mathrm{zt}}
$$

and thus each $W_{\sigma}[i, j]$ is a zero-tested CLT13-encoding of 0 .

Following the intuition from Section 2.3.3, we can write

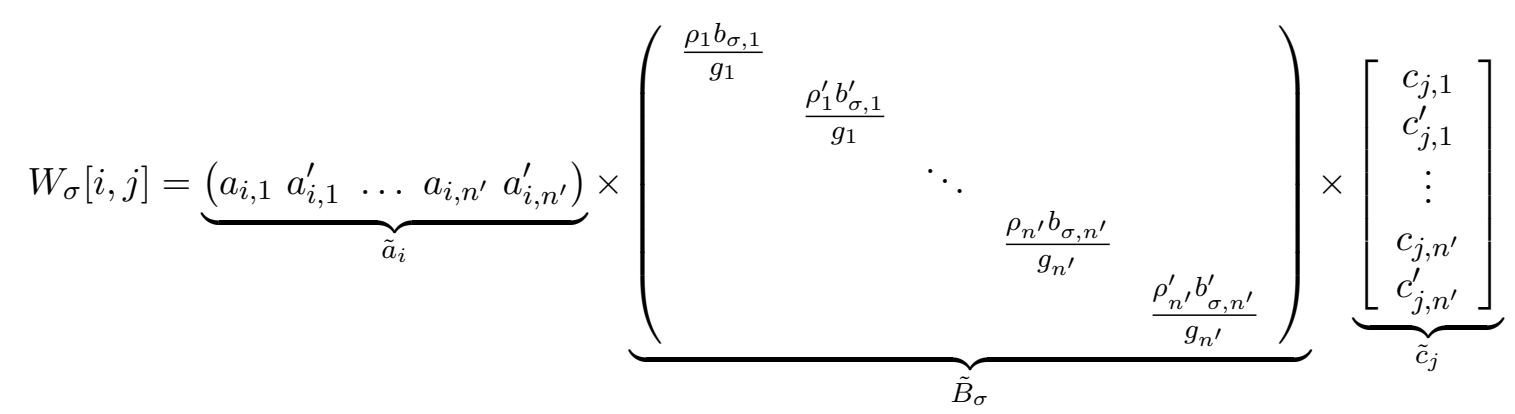

where the $g_{i}$ 's are set by the CLT13 scheme and the remaining values are set as follows.

Each $a_{i, k}\left(k \in\left[n^{\prime}\right]\right)$ is the numerator of the $k$ th CRT component of $\hat{a}_{i}$. That is, $a_{i, k}=g_{k} r_{a, i, k}+$ $\alpha_{i, k}$ for independent randomness $r_{a, i, k}$, where $\hat{a}_{i}$ encodes plaintext $\left(\alpha_{i, k}\right)_{k \in\left[n^{\prime}\right]}$. Similarly $a_{i, k}^{\prime}, b_{\sigma, k}$, $b_{\sigma, k}^{\prime} c_{j, k}$ and $c_{j, k}^{\prime}$ are the $k$ th numerators of their respective encodings. Finally the $\rho_{k}$ 's contain the remaining "system parameters", which here include the CRT components of $\hat{t}_{L}$ and $\hat{t}_{R}$. Namely, $\rho_{k}=p_{k}^{*} h_{k} t_{L, k}$ and $\rho_{k}^{\prime}=-p_{k}^{*} h_{k} t_{R, k}$, where $t_{L, k}$ and $t_{R, k}$ are the $k$ th numerators of $\hat{t}_{L}$ and $\hat{t}_{R}$ respectively. Note that this equation holds over $\mathbb{Q}$, without reducing $\bmod x_{0}$, because the encodings are chosen so that $a_{i, k} b_{\sigma, k} c_{j, k} t_{L, k}-a_{i, k}^{\prime} b_{\sigma, k}^{\prime} c_{j, k}^{\prime} t_{R, k}$ is a small multiple of $g_{k}$ for every $i, j, k, \sigma$.

Now, for $\sigma \in\{0,1\}$, we can write $W_{\sigma}=\tilde{A} \times \tilde{B}_{\sigma} \times \tilde{C}_{j}$ where $\tilde{A}$ has $i$ th row $\tilde{a}_{i}$ and $\tilde{C}$ has $j$ th column $\tilde{c}_{j}$. Each $W_{\sigma}$ is invertible over $\mathbb{Q}$ with high probability, as follows. Clearly $\tilde{B}_{\sigma}$ is invertible. To see that $\tilde{A}$ is invertible, consider its determinant as a non-trivial degree- $n$ polynomial in the randomizers $r_{a, i, k}$. Since these are $\operatorname{neg}(n)$-close to uniform over a suitably large field by Lemma 3.2, $\tilde{A}^{\prime}$ 's determinant will be non-zero over $\mathbb{Q}$ with high probability by the Schwartz-Zippel lemma. The same argument shows $\tilde{C}$ is invertible with high probability. Thus we can write $W:=W_{0} \times W_{1}^{-1}=\tilde{A} \times \tilde{B}_{0} \times \tilde{B}_{1}^{-1} \times \tilde{A}^{-1}$.

To prove the first property of Definition 1, we observe that

$$
\begin{aligned}
\operatorname{charPoly}(W)=\operatorname{charPoly}\left(\tilde{B}_{0} \times \tilde{B}_{1}^{-1}\right) & =\prod_{k \in\left[n^{\prime}\right]}\left(\lambda-\frac{b_{0, k}}{b_{1, k}}\right)\left(\lambda-\frac{b_{0, k}^{\prime}}{b_{1, k}^{\prime}}\right) \\
& =\prod_{k \in\left[n^{\prime}\right]} \operatorname{charPoly}\left(\left(B_{0} \bmod p_{k}\right) \times\left(B_{1} \bmod p_{k}\right)^{-1}\right) .
\end{aligned}
$$

where $\lambda$ is the characteristic polynomial's indeterminate.

To prove the second property of Definition 1, we consider each $\operatorname{det}\left(\left[B_{1}\right]_{p_{k}}\right)$ as a polynomial over the randomizers $r_{b, 1, k}$. By Lemma 3.2 and the Schwartz-Zippel lemma, we have $\operatorname{det}\left(\left[B_{1}\right]_{p_{k}}\right) \neq$ $0\left(\bmod p_{k}\right)$ with probability $1-\operatorname{neg}(n)$. Then $B_{1}$ is non-singular $\bmod p_{k}$ for all $k \in[n]$ simultaneously with probability $1-\operatorname{negl}(n)$, and thus it is non-singular also mod $x_{0}$.

To prove the third property of Definition 1, we observe that any value $d_{k}$ in step 4 of Figure 1 is either $b_{1, k}$ or $b_{1, k}^{\prime}$ for some $k \in\left[n^{\prime}\right]$, and these are all co-prime to $x_{0}$. 
Proving the fourth property of Definition 1 reduces to showing that, for every $k \in\left[n^{\prime}\right]$, both $\left(b_{1, k} \lambda-b_{0, k}\right)$ and $\left(b_{1, k}^{\prime} \lambda-b_{0, k}^{\prime}\right)$ are non-zero modulo $p_{k}$ when setting $\lambda=B_{0} \times B_{1}^{-1}\left(\bmod x_{0}\right)$. This again holds with high probability over the CLT randomizers.

We remark that this attack can be extended to break the assumption of Gentry et al. [14] even when their encodings are transformed using the above technique from [4]. In general, for this attack on [4], we only require encodings that "BWZ-multiply" to 0 at the "BWZ-zero-testing-level"; thus any set of encodings susceptible to the "basic" attack will be susceptible to the attack here after applying the 4] transformation.

\subsection{Attacking the Garg-Gentry-Halevi-Zhandry Countermeasure}

Garg, Gentry, Halevi, and Zhandry proposed in [12, Sec. 7] a variant of the CLT13 scheme, that was designed to resist the CHLRS attack. This variant uses matrices of native CLT13 encodings, where the encoded value is an eigenvalue of the matrix and the zero-test parameter includes also the corresponding eigenvector. The CHLRS attack from [6] indeed does not apply to this variant, but below we show that this variant still gives rise to a good attack set, and thus our new attack from Figure 1 recovers the secret primes.

The GGHZ variant relies on the same parameters as CLT13, namely we choose $\left(\left\{g_{i}\right\}_{i},\left\{p_{i}\right\}_{i}, \mathbf{p}_{\mathrm{zt}},\left\{z_{i}\right\}\right)$ (with $x_{0}:=\prod_{i} p_{i}$ and top level corresponding to denominator $z^{*}=\prod z_{i}$ ). Let $d:=2 \kappa+1$, and choose a secret matrix $T \in \mathbb{Z}_{x_{0}}^{d \times d}$ uniformly. An encoding of a plaintext value $c$ at some level is given by $C \in \mathbb{Z}_{x_{0}}^{d \times d}$, where 4

$$
C:=T \times \underbrace{\left[\begin{array}{cccc}
\hat{\$} & \hat{0} & \cdots & \hat{0} \\
\hat{0} & \hat{\$} & \cdots & \hat{0} \\
\vdots & & & \vdots \\
\hat{0} & \hat{0} & \cdots & \hat{c}
\end{array}\right]}_{C^{*}} \times T^{-1} \bmod x_{0} .
$$

Each $\hat{\$}$ in $C^{*}$ is a "native CLT13 encoding" of an independent random value at the given level, each $\hat{0}$ is an independent native encoding of 0 , and $\hat{c}$ is a native encoding of $c$. For zero-testing, two dimension- $d$ vectors $s, t$ are provided:

$$
\begin{array}{lll}
s:= & {[\hat{\$} \ldots \hat{\$} \hat{0} \ldots \hat{0} \hat{\$}] \times T^{-1}} & \bmod x_{0} \\
t:=\mathbf{p}_{\text {zt }} \cdot T \times & {[\hat{0} \ldots \hat{0} \hat{\$} \ldots \hat{\$} \hat{\$}]^{T}} & \bmod x_{0}
\end{array}
$$

where $\hat{0}$ and $\hat{\$}$ are CLT13 native "level-zero" encodings (i.e, corresponding to denominator 1$)$. Then a GGHZ-encoding $C$ as above at the top level level can be zero tested by computing $s \times C \times t=$ $(\hat{\$} \cdot \hat{c}+\hat{0}) \cdot \mathbf{p}_{\mathrm{zt}}\left(\bmod x_{0}\right)$ and checking for smallness.

Attack set. The matrix sets $\mathcal{A}, \mathcal{B}, \mathcal{C}$ consist directly of GGHZ-encodings, since these are already in matrix form. Specifically, we assume that $[1, \kappa]$ is partitioned into three intervals $I_{A}=\left[1, k_{A}\right], I_{B}=$ $\left[k_{A}+1, k_{B}\right], I_{C}=\left[k_{B}+1, \kappa\right]$, such that we have GGHZ-encodings

\footnotetext{
${ }^{4}$ The attack applies also when one uses many matrices $T_{0}, T_{0}^{-1}, \ldots, T_{\kappa}, T_{\kappa}^{-1}$ (rather than just $T, T^{-1}$ ), so multiplication can only be performed in a specific order, as described in [12].
} 
- $\mathcal{A}=\left\{A_{i}=T \times A_{i}^{*} \times T^{-1}: A_{i} \text { encoded at level } I_{A}\right\}_{i \in[n d]}$

- $\mathcal{B}=\left\{B_{\sigma}=T \times B_{\sigma}^{*} \times T^{-1}: B_{\sigma} \text { encoded at level } I_{B}\right\}_{\sigma \in\{0,1\}}$

- $\mathcal{C}=\left\{C_{k}=T \times C_{k}^{*} \times T^{-1}: C_{k} \text { encoded at level } I_{C}\right\}_{k \in[n d]}$

where $A_{i} \times B_{\sigma} \times C_{k}$ is a GGHZ-encoding of 0 for all $i, k \in[n d]$ and $\sigma \in\{0,1\}$. The vectors $s$ and $t$ are the zero testing vectors from the GGHZ scheme.

Attack set properties. We prove that $(\mathcal{A}, \mathcal{B}, \mathcal{C}, s, t)$ form a good attack set according to Definition 1. We write

$$
\begin{aligned}
W_{\sigma}[i, k] & =s \times A_{i} \times B_{\sigma} \times C_{k} \times t \\
& =s \times T \times A_{i}^{*} \times B_{\sigma}^{*} \times C_{k}^{*} \times T^{-1} \times t=\mathbf{a}^{i} \times B_{\sigma}^{*} \times \mathbf{c}^{k}
\end{aligned}
$$

where $\mathbf{a}^{i}:=s^{\prime} \times A_{i}^{*}$ and $\mathbf{c}^{k}:=C_{k}^{*} \times t^{\prime}$ are dimension- $d$ vectors. The above equality holds over the integers, not only modulo $x_{0}$, since all the variables in the final right-hand-side are small compared to $x_{0}$.

We denote $\mathbf{a}_{\ell}^{i}:=\mathbf{a}^{i} \bmod p_{\ell}$ and $\mathbf{c}_{\ell}^{k}:=\mathbf{c}^{k} \bmod p_{\ell}$ for $i \in[n d], \ell \in[n]$. Now we can write $W_{\sigma}=\tilde{A} \times \tilde{B}_{\sigma} \times \tilde{C}$, where $\tilde{A}$ is an $n d \times n^{2} d$ matrix, $\tilde{C}$ is an $n^{2} d \times n d$ matrix, and $\tilde{B}_{\sigma}$ is a $n^{2} d \times n^{2} d$ block-diagonal matrix, defined as follows.

$$
\begin{gathered}
\tilde{A}=\left[\begin{array}{cccc}
\mathbf{a}_{1}^{1} & \mathbf{a}_{2}^{1} & \cdots & \mathbf{a}_{n}^{1} \\
\mathbf{a}_{1}^{2} & \mathbf{a}_{2}^{2} & \cdots & \mathbf{a}_{n}^{2} \\
\vdots & \vdots & & \vdots \\
\mathbf{a}_{1}^{n d} & \mathbf{a}_{2}^{n d} & \cdots & \mathbf{a}_{n}^{n d}
\end{array}\right] \quad \tilde{C}=\left[\begin{array}{cccc}
\left(\mathbf{c}_{1}^{1}\right)^{T} & \left(\mathbf{c}_{1}^{2}\right)^{T} & \cdots & \left(\mathbf{c}_{1}^{n d}\right)^{T} \\
\left(\mathbf{c}_{2}^{1}\right)^{T} & \left(\mathbf{c}_{2}^{2}\right)^{T} & \cdots & \left(\mathbf{c}_{2}^{n d}\right)^{T} \\
\vdots & \vdots & & \vdots \\
\left(\mathbf{c}_{n}^{1}\right)^{T} & \left(\mathbf{c}_{n}^{2}\right)^{T} & \cdots & \left(\mathbf{c}_{n}^{n d}\right)^{T}
\end{array}\right] \\
\tilde{B}_{\sigma}=\left[\begin{array}{ccccc}
B_{\sigma}^{*} \bmod p_{1} & 0 & & 0 \\
0 & B_{\sigma}^{*} \bmod p_{2} & & 0 \\
& & \ddots & \\
0 & 0 & & B_{\sigma}^{*} \bmod p_{n}
\end{array}\right]
\end{gathered}
$$

Using Lemma 3.2 and the Schwartz-Zippel lemma, it can be shown that with high probability over the randomness in the CLT13 encodings, $\tilde{A}, \tilde{C}$, and each $B_{\sigma}^{*}$ have full rank $n d$. Under this condition each $W_{\sigma}$ has rank $n d$ and is thus invertible, so we can write $W=W_{0} \times W_{1}^{-1}=\tilde{A} \times \tilde{B}_{0} \times \tilde{B}_{1}^{-1} \times \tilde{A}^{-1}$, where $\tilde{A}^{-1}$ denotes the right inverse of the (non-square, full-rank) matrix $\tilde{A}$. Then we have

$$
\begin{aligned}
\operatorname{charPoly}(W)=\operatorname{charPoly}\left(\tilde{B}_{0} \times \tilde{B}_{1}^{-1}\right) & =\prod_{i=1}^{n} \operatorname{charPoly}\left(\left[B_{0}^{*}\right]_{p_{i}} \times\left[B_{1}^{*}\right]_{p_{i}}^{-1}\right) \\
& =\prod_{i=1}^{n} \operatorname{charPoly}\left(\left[B_{0}\right]_{p_{i}} \times\left[B_{1}\right]_{p_{i}}^{-1}\right)
\end{aligned}
$$

so the first property of Definition 1 holds. The second property of Definition 1 follows similarly to the proof for the BWZ attack (Section 3.2). We were not able to prove that the last two properties 
in Definition 1 hold, but we verified them experimentally by running the attack on several random instances and checking that they indeed hold in all of them. For the fourth property, we can prove that it holds under the following natural conjecture:

Conjecture 1. For each $i \in[n]$, with high probability over the randomness in the CLT13 encodings, charPoly $\left(\left[B_{0}^{*}\right]_{p_{i}} \times\left[B_{1}^{*}\right]_{p_{i}}^{-1}\right)$ is irreducible over $\mathbb{Q}$.

We make two remarks about this conjecture. First, we have verified it experimentally. Second, a work of Kuba [19] shows that among the degree- $n$ univariate integer polynomials whose coefficients are bounded in absolute value by an integer $t$, the polynomials that are reducible over $\mathbb{Q}$ make up a roughly $1 / t$ fraction. In particular, a random polynomial with $r$-bit coefficients is irreducible over $\mathbb{Q}$ with probability roughly $1-2^{-r}$. Thus provided that charPoly $\left(\left[B_{0}^{*}\right]_{p_{i}} \times\left[B_{1}^{*}\right]_{p_{i}}^{-1}\right)$ is welldistributed among polynomials with an appropriate coefficient bound, Conjecture 1 should hold. We note that the relationship between a random polynomial and the characteristic polynomial of a random matrix has been explored by Hansen and Schmutz [17. However, their results do not seem directly applicable here because they study polynomials over a finite field $\mathbb{F}$, and a uniform degree- $n$ polynomial is irreducible over $\mathbb{F}$ only with probability $\approx 1 / n$.

Assuming Conjecture 1, the fourth property of Definition 1 reduces to showing that for every prime factor $p_{j}$ of $x_{0},\left(\prod_{i \neq j} d_{i} f_{i}\right)(M) \neq 0\left(\bmod p_{j}\right)$ where $d_{i}, f_{i}$, and $M$ are as in Fig. 1. Choose all values in the CLT13 encodings except for the random values in the $j$ th slot of the encodings in $B_{0}$, and call the unchosen values $R$. With high probability over this choice, each entry of $M$ is a non-trivial linear polynomial in $R$, and $\left(\prod_{i \neq j} d_{i} f_{i}\right)$ is a non-trivial degree- $(n-1)$ polynomial in $M$. Thus each entry of $\left(\prod_{i \neq j} d_{i} f_{i}\right)(M)$ is a non-trivial degree- $(n-1)$ polynomial in $R$, and is non-zero modulo $p_{i}$ with high probability by Lemma 3.2 and the Schwartz-Zippel lemma.

\subsection{Attacking GGHRSW Obfuscation for Simple Branching Programs}

We observe that our unified attack can be applied also to the candidate obfuscation construction of Garg et al. [11] when instantiated with the CLT13 multilinear maps and applied to branching programs with specific "partitionable" structure that we define below. We stress that applying Barrington's theorem to a circuits does not have the required structure, so as far as we know, the iO candidate from [11] for $N C^{1}$ circuits remains plausible.

\section{The GGHRSW Obfuscation Candidate for Branching Programs}

Recall that the obfuscator of Garg et al. [11] consists of encoded, randomized versions of two BPs; one is the BP that we want to obfuscate and the other is a "dummy BP" consisting of only identity matrices (and hence computing the all-one function). Even though neither program computes a zero, they are constructed such that their difference on accepting computations yields an encoding of zero, which can be recognized by zero testing. The core construction from [11] works with oblivious branching programs. An oblivious branching program of length $L$ over $\ell$ input variables is defined as follows

$$
B P=\left\{\left(\operatorname{inp}(i), A_{i, 0}, A_{i, 1}\right): i \in[L], \operatorname{inp}(i) \in[\ell], A_{i, b} \in\{0,1\}^{w \times w}\right\},
$$

where the $A_{i \sigma}$ 's are invertible matrices and $\operatorname{inp}(i)$ is the input bit position examined in step $i$. The function computed by this branching program is defined (using some fixed matrix $A_{0} \neq I$ ) as 


$$
f_{B P, A, I}= \begin{cases}0 & \text { if } \prod_{i=1}^{L} A_{i, x_{\mathrm{inp} i}}=A_{0} \\ 1 & \text { if } \prod_{i=1}^{L} A_{i, x_{\mathrm{inp} i}}=I \\ \text { undef } & \text { otherwise. }\end{cases}
$$

Let $\mathbb{Z}_{p}$ be a ring that we use for randomization, and for each input bit $j$ denote by $I_{j}:=\{i \in$ $[L]: \operatorname{inp}(i)=j\}$ the set of steps where the branching program examine the $j$ 'th input bit. The GGHRSW construction, on input an $L$-step branching program $B P$ over $\ell$ input bits, proceeds as follows:

1. Sample random and independent scalars $\left\{\alpha_{i, 0}, \alpha_{i, 1}, \alpha_{i, 0}^{\prime}, \alpha_{i, 1}^{\prime} \in \mathbb{Z}_{p}: i \in[L]\right\}$, subject to the constraint that for any input bit $j \in[\ell]$, we have $\prod_{i \in I_{j}} \alpha_{i, 0}=\prod_{i \in I_{j}} \alpha_{i, 0}^{\prime}$ and $\prod_{i \in I_{j}} \alpha_{i, 1}=$ $\prod_{i \in I_{j}} \alpha_{i, 1}^{\prime}$.

2. Let $m=2 L+w$. For every $i \in[n]$, choose two block-diagonal $m \times m$ matrices $D_{i, 0}, D_{i, 1}$ where the diagonal entries $1, \ldots, 2 L$ are chosen at random $(\$)$ and the bottom-right $w \times w$ are the scaled $A_{j, b}$ 's. Also choose two more $m \times m$ matrices $D_{i, 0}^{\prime}, D_{i, 1}^{\prime}$ where the diagonal entries $1, \ldots, 2 L$ are random and the bottom-right $w \times w$ are the scaled identity:

$$
D_{i, b} \sim\left(\begin{array}{cccc}
\$ & & & \\
& \ddots & & \\
& & \$ & \\
& & & \alpha_{i, b} A_{i, b}
\end{array}\right), D_{i, b}^{\prime} \sim\left(\begin{array}{cccc}
\$ & & \\
& \ddots & \\
& & \$ & \\
& & \alpha_{i, b}^{\prime} I
\end{array}\right), b \in\{0,1\} .
$$

3. Choose vectors $\mathbf{s}$ and $\mathbf{t}$, and $\mathbf{s}^{\prime}$ and $\mathbf{t}^{\prime}$ of dimension $m=2 L+w$ as follows:

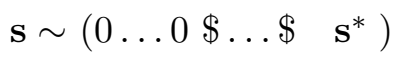

$$
\begin{aligned}
& \mathbf{t} \sim\left(\begin{array}{lllll}
\$ \ldots & \ldots & \ldots 0 & \mathbf{t}^{*}
\end{array}\right)^{T} \\
& \mathbf{s}^{\prime} \sim\left(0 \ldots 0 \$ \ldots \$ \quad \mathbf{s}^{\prime *}\right) \\
& \mathbf{t}^{\prime} \sim\left(\begin{array}{lllll}
\$ & \ldots & \ldots & \ldots 0 & \mathbf{t}^{*}
\end{array}\right)^{T}
\end{aligned}
$$

Here $\mathbf{s}^{*}, \mathbf{t}^{*}, \mathbf{s}^{\prime *}, \mathbf{t}^{\prime *} \in \mathbb{Z}_{p}^{w}$ are uniform up to $\left\langle\mathbf{s}^{*}, \mathbf{t}^{*}\right\rangle=\left\langle\mathbf{s}^{\prime *}, \mathbf{t}^{\prime *}\right\rangle$, and $0 \ldots 0$ and $\$ \ldots \$$ are length- $L$ vectors of zeros and uniform elements of $\mathbb{Z}_{p}$, respectively.

4. Sample $2(L+1)$ uniform full-rank matrices $R_{0}, \ldots, R_{L}, R_{0}^{\prime}, \ldots, R_{L}^{\prime} \in \mathbb{Z}_{p}^{m \times m}$.

5. The randomized branching program over $\mathbb{Z}_{p}$ is the following:

$$
\begin{aligned}
& \mathcal{R N}_{\mathcal{N}}(B P)= \\
& \left\{\begin{array}{ll}
\tilde{\mathbf{s}}=\mathbf{s} R_{0}^{-1}, \tilde{\mathbf{t}}=R_{n} \mathbf{t}, & \tilde{\mathbf{s}}^{\prime}=\mathbf{s}^{\prime}\left(R_{0}^{\prime}\right)^{-1}, \tilde{\mathbf{t}}^{\prime}=R_{n}^{\prime} \mathbf{t}^{\prime} \\
\left\{\tilde{D}_{i, b}=R_{i-1} D_{i, b} R_{i}^{-1}\right\}_{i \in[L], b \in\{0,1\}}, & \left\{\tilde{D}_{i, b}^{\prime}=R_{i-1}^{\prime} D_{i, b}^{\prime}\left(R_{i}^{\prime}\right)^{-1}\right\}_{i \in[L], b \in\{0,1\}}
\end{array}\right\}
\end{aligned}
$$

6. Finally, encode the randomized program using an $(L+2)$-level asymmetric multilinear map scheme. Here we use the CLT13 scheme, choosing $x_{0}=\prod_{i=1}^{n} p_{i}$, for equal-size primes $p_{i}, g=$ CRT $\left(g_{i}\right)$ for small $g_{i} \ll p_{i}$ 's, random denominators $z_{0}, z_{1}, \ldots, z_{L+1} \in \mathbb{Z}_{x_{0}}$ with $z^{*}=\left[\prod_{i} z_{i}\right]_{x_{0}}$, and an element $h$ with mid-size CRT components, used for the zero-testing parameter $\mathbf{p}_{\mathrm{zt}}=$ $\left[h z^{*} g^{-1}\right]_{x_{0}}$. 
Choose random small vectors $\mathbf{r}_{\mathbf{s}} \mathbf{r}_{\mathbf{s}}^{\prime} \mathbf{r}_{\mathbf{t}} \mathbf{r}_{\mathbf{t}}^{\prime}$, and random small matrices $U_{i, b}$ and $U_{i, b}^{\prime}$, and publish the zero-testing parameter $\mathbf{p}_{\mathrm{zt}}$ and the obfuscation

$$
\mathcal{O}(B P)=\left\{\begin{array}{l}
\hat{\mathbf{s}}=\left[z_{0}^{-1}\left(\tilde{\mathbf{s}}+g \mathbf{r}_{\mathbf{s}}\right)\right]_{x_{0}}, \quad \hat{\mathbf{t}}=\left[z_{L+1}^{-1}\left(\tilde{\mathbf{t}}+g \mathbf{r}_{\mathbf{t}}\right)\right]_{x_{0}}, \\
\left\{\hat{D}_{i, b}=\left[z_{i}^{-1}\left(\tilde{D}_{i, b}+g U_{i, b}\right)\right]_{x_{0}}\right\}_{i \in[L], b \in\{0,1\}} \\
\hat{\mathbf{s}}^{\prime}=\left[z_{0}^{-1}\left(\tilde{\mathbf{s}}^{\prime}+g \mathbf{r}_{\mathbf{s}}^{\prime}\right)\right]_{x_{0}}, \quad \hat{\mathbf{t}}^{\prime}=\left[z_{L+1}^{-1}\left(\tilde{\mathbf{t}}^{\prime}+g \mathbf{r}_{\mathbf{t}}^{\prime}\right)\right]_{x_{0}}, \\
\left\{\hat{D}_{i, b}^{\prime}=\left[z_{i}^{-1}\left(\tilde{D}_{i, b}^{\prime}+g U_{i, b}^{\prime}\right)\right]_{x_{0}}\right\}_{i \in[L], b \in\{0,1\}}
\end{array}\right\} .
$$

To evaluate $\mathcal{O}(B P)(x)$, compute $y=\tilde{s}\left(\prod_{i=1}^{L} \tilde{D}_{i, x_{\operatorname{inp}(i)}}\right) \tilde{t}-\tilde{s}^{\prime}\left(\prod_{i=1}^{L} \tilde{D}_{i, x_{\operatorname{inp}(i)}}^{\prime}\right) \tilde{t}^{\prime}$, and output 1 if $y$ encodes 0 (as determined by $\mathbf{p}_{\mathrm{zt}}$ ).

\section{Attack}

Our attack is applicable to branching programs with the following structure: there exists a partition of the input bits $[\ell]=X_{1} \cup X_{2} \cup X_{3}$ and the branching program steps $[L]=A \cup B \cup C$ such that $A, B$ and $C$ consist of consecutive steps in the branching program and $\operatorname{inp}(i) \in X_{1} \forall i \in A, \operatorname{inp}(i) \in$ $X_{2} \forall i \in B$ and $\operatorname{inp}(i) \in X_{3} \forall i \in C$. We consider a branching program $B P$ of length $L$ and input length $\ell$, computing the constant-1 function, that can be written as $B P(x)=A\left(x_{1}\right) \circ B\left(x_{2}\right) \circ C\left(x_{3}\right)$, where $A\left(x_{1}\right), B\left(x_{2}\right)$, and $C\left(x_{3}\right)$ are branching programs over positions in the sets $A, B$, and $C$ depending on inputs $x_{1}, x_{2}$, and $x_{3}$, respectively. We are given the obfuscation:

$$
\mathcal{O}(B P)=\left(\mathbf{p}_{\mathrm{zt}}, \hat{\mathbf{s}}, \hat{\mathbf{t}}, \hat{\mathbf{s}}^{\prime}, \hat{\mathbf{t}}^{\prime},\left\{\hat{D}_{i, b}, \hat{D}_{i, b}^{\prime}\right\}_{i \in[L], b \in\{0,1\}}\right) .
$$

Attack Sets. We construct the sets $\mathcal{A}, \mathcal{B}$ and $\mathcal{C}$ as follows. Let $A(x)=\prod_{i \in A} D_{i, x_{\text {inp }(i)}}, A^{\prime}(x)=$ $\prod_{i \in A} D_{i, x_{\text {inp }(i)}}^{\prime}$. We define similarly $B(x), B^{\prime}(x)$ and $C(x), C^{\prime}(x)$. We note that using $\mathcal{O}$ we can compute $R_{0} A(x) R_{|A|}^{-1}=\prod_{i \in A} \tilde{D}_{i, x_{\operatorname{inp}(i)}}$ and $R_{0} A^{\prime}(x) R_{|A|}^{-1}=\prod_{i \in A} \tilde{D}_{i, x_{\text {inp }(i)}}^{\prime}$, and so on. Let $\alpha_{1}, \ldots, \alpha_{m n} \in$ $\{0,1\}^{\left|X_{1}\right|}$ be any set of distinct strings, and similarly for $\beta_{0}, \beta_{1} \in\{0,1\}^{\left|X_{2}\right|}$ and $\gamma_{1}, \ldots, \gamma_{m n} \in$ $\{0,1\}^{\left|X_{3}\right|}$. We set $s=\left(\tilde{s}, \tilde{s}^{\prime}\right)$ and $t=\left(\tilde{t},-\tilde{t}^{\prime}\right) \mathbf{p}_{\mathrm{zt}}$, and define

$$
\begin{aligned}
& \mathcal{A}=\left\{\tilde{A}_{i}=\left[\begin{array}{cc}
R_{0} A\left(\alpha_{i}\right) R_{|A|}^{-1} & 0 \\
0 & R_{0} A^{\prime}\left(\alpha_{i}\right) R_{|A|}^{-1}
\end{array}\right]\right\}_{i \in[(2 L+w) n]} \\
& \mathcal{B}=\left\{\tilde{B}_{\sigma}=\left[\begin{array}{cc}
R_{|A|} B\left(\beta_{\sigma}\right) R_{|A \cup B|}^{-1} & 0 \\
0 & R_{|A|} B^{\prime}\left(\beta_{\sigma}\right) R_{|A \cup B|}^{-1}
\end{array}\right]\right\}_{\sigma \in\{0,1\}} \\
& \mathcal{C}=\left\{\tilde{C}_{k}=\left[\begin{array}{cc}
R_{|A \cup B|} C\left(\gamma_{k}\right) R_{L}^{-1} & 0 \\
0 & R_{|A \cup B|} C^{\prime}\left(\gamma_{k}\right) R_{L}^{-1}
\end{array}\right]\right\}_{k \in[(2 L+w) n]} .
\end{aligned}
$$

Set Properties. We consider the values

$$
W_{0}[i, k]=s \times \tilde{A}_{i} \times \tilde{B}_{0} \times \tilde{C}_{k} \times t=\left(s \times A_{i} \times B_{0} \times C_{k} \times t-s^{\prime} \times A_{i}^{\prime} \times B_{0}^{\prime} \times C_{k}^{\prime} \times t^{\prime}\right) \mathbf{p}_{\mathrm{zt}} .
$$


Since $W_{0}[i, k]$ is a zero-tested encoding of zero by the definition of the obfuscated branching programs, the above equality holds not only $\bmod x_{0}$ but also over the integers. $W_{1}$ is constructed analogously.

The rest of the attack proceeds in the same manner as the attack on GGHZ encodings from Section 3.3. Let $\mathbf{a}_{i}=\left(s \times A_{i}, s^{\prime} \times A_{i}^{\prime}\right)$ for $i \in[(2 m+w) n], \mathbf{c}_{k}=\left(C_{k} \times t \times \mathbf{p}_{\mathrm{zt}},-C_{k}^{\prime} \times t^{\prime} \times \mathbf{p}_{\mathrm{zt}}\right)$ for

$k \in[(2 m+w) n]$ and $X_{0}=\left[\begin{array}{cc}B_{0} & 0 \\ 0 & B_{0}^{\prime}\end{array}\right]$. We set the matrix $\hat{A}$ to have $i$-th row that is concatenations of the vectors $\mathbf{a}_{i} \bmod p_{j}$ for $j \in[n]$, the matrix $\hat{C}$ to have $i$-th column that is concatenation of $\mathbf{c}_{i}^{T} \bmod p_{j}$ for $j \in[n]$, and the matrix $\hat{B}_{0}$ to be a diagonal matrix with diagonal consisting of $X_{0} \bmod p_{j}$ for $j \in[n]$. Then we have that $W_{0}=\hat{A} \times \hat{B}_{0} \times \hat{C}$. We compute analogously $W_{1}=\hat{A} \times \hat{B}_{1} \times \hat{C}$. We use these matrices as in the attack on GGHZ encodings to break the underlying CLT13 encodings.

\subsection{Attacking Recent Circuit-Obfuscation Schemes}

Recently, Zimmerman 22] and Applebaum and Brakerski [1] proposed very similar obfuscation schemes that handle circuits directly, without needing to transform them first into branching programs. This scheme operates with MMAP schemes that have composite-order plaintext space, it was proved secure in a generic MMAPs model, and at the time it was suggested to realize this scheme over the CLT13 graded encoding scheme [7]. Although the attacks of Cheon et al. serve as a powerful demonstration that the CLT13 scheme is susceptible to a lot more attacks than those available in the generic model, it was still not known how to apply these attacks to break the schemes from [22, 1].

Each of the works [22, 1] contain a "simple scheme" (which is essentially the same in both works) and then a more complicated variant (which differs between the two papers). Below we show that the common simple scheme from [22, Appendix A] and [1] can be broken using an attack similar to the one from 3.2, when instantiated with simple enough circuits. For example, when that scheme is used to obfuscate point functions, it is possible to recover the secret point from the obfuscated circuit.

\section{The Circuit-Obfuscation Scheme}

We begin with an overview of the scheme from [22, Appendix A]. We omit some details which are not relevant to the attacks.

The construction uses a "universal circuit" $C(y, x)=C_{y}(x), C:\{0,1\}^{k} \times\{0,1\}^{m} \rightarrow\{0,1\}$. The starting point of this construction is the following simple scheme: obfuscating a circuit $C_{y}$ is done by encoding the bits of $y$ and $x$ using a graded encoding scheme, providing for the bits of $y$ only encoding of the correct bit value ( 0 or 1 ) and for the bits of $x$ the encoding of both 0 and 1 . The user can then compute $C(y, x)$ on the encoded bits and check if the answer is zero using the zero-test. Of course this scheme is insecure, and so several modifications are introduced:

- The scheme uses a composite order multi-linear maps scheme that allows each encoding to have two slots relative to two different factors $N_{\text {ev }}, N_{c h k}$ of the plaintext space. One slot encodes the bits as before (call it the signal slot and is defined by the value modulo $\mathrm{N}_{\mathrm{ev}}$, while the other slot encodes random elements (call it the control slot) and is defined by the value modulo $\mathrm{N}_{\text {chk }}$. An encoding of a message $m$ such that $m=m_{1} \bmod \mathrm{N}_{\mathrm{ev}}$ and $=m_{2} \bmod \mathrm{N}_{\text {chk }}$ with respect to a set $S$ is denoted as $\left[m_{1}, m_{2}\right]_{S}$. 
For each bit position $i$ in $x$, we provide two encodings $\tilde{x}_{i, 0}=\left[0, \alpha_{i}\right]_{X_{i, 0}}$ and $\tilde{x}_{i, 1}=\left[1, \alpha_{i}\right]_{X_{i, 1}}$ with the same value $\alpha_{i}$ in the control slot and values zero and one in the signal slot. Similarly for each bit position $j$ in $y$ we encode a random element $\beta_{j}$ in the control slot together with the value $y_{i}$ in the signal slot: $\tilde{y}_{i}=\left[y_{i}, \beta_{i}\right]_{Y}$. The encodings of the bits belonging to the function description $y$ use the same set $Y$, while the encoding of each input bit $b$ at each position $i$ of $x$ uses a different set $X_{i, b}$.

The obfuscation includes the above encodings $\tilde{y}_{i}$ and $\tilde{x}_{j}$ together with an encoding of

$$
\tilde{C}^{*}=\left[0, C^{*}\right]_{Y^{\operatorname{deg}(y)}} \prod_{i \in[n]}\left(X_{i, 0} X_{i, 1}\right)^{\operatorname{deg}\left(x_{i}\right)} Z_{i},
$$

where $C^{*}$ is computed as $C\left(\alpha_{1}, \ldots, \alpha_{k}, \beta_{1}, \ldots, \beta_{m}\right), \operatorname{deg}(y)$ is the total degree of $C$ in all of the variables $y_{1}, \ldots, y_{m}$ and $\operatorname{deg}\left(x_{i}\right)$ is the total degree of the $C$ in the variable $x_{i}$. The sets $Z_{i}$ are new sets, which are used in the straddling encoding sets as we explain in the next paragraph.

To evaluate the program on any specific input $x$, we choose the appropriate encodings $\tilde{x}_{i, x_{i}}$ together with the encodings $\tilde{y}_{i}$ and use them to evaluate the circuit $C$ and obtain $\tilde{C}=$ $\left[C_{y}(x), C^{*}\right]_{Y^{\operatorname{deg}(y)}} \prod_{i \in[k]}\left(X_{i, x_{i}}\right)^{\operatorname{deg}\left(x_{i}\right)}$. In order apply the zero test we need to remove the value in the control slot of the encoding. For this purpose we will subtract the encoding $\tilde{C}^{*}$. However, since $\tilde{C}$ and $\tilde{C}^{*}$ are encoded at different levels, the obfuscation provides the following additional encodings:

$$
\tilde{z}_{i, b}=\left[\delta_{i, b}, \gamma_{i, b}\right]_{X_{i, 1-b}^{\operatorname{deg}\left(x_{i}\right)} Z_{i} W_{i}} \quad \tilde{w}_{i, b}=\left[0, \gamma_{i, b}\right]_{W_{i}} .
$$

These encodings extend the straddling sets to enforce guarantees that each input bit is used consistently throughout the circuit. More specifically $\tilde{C}$ can be completed to the zero-testing level using the $\tilde{z}_{i, b}$ encodings if and only if it is encoded at a level that has component either $X_{i, 0}^{\operatorname{deg}\left(x_{i}\right)}$ or $X_{i, 1}^{\operatorname{deg}\left(x_{i}\right)}$.

Using the above encodings we can compute the following encoding at zero-testing level:

$$
z=\left(\tilde{C} \prod_{i \in[n]} \tilde{z}_{i, x_{i}}-\tilde{C}^{*} \prod_{i \in[n]} \tilde{w}_{i, x_{i}}\right) .
$$

We can apply the zero test on $z$, which encodes 0 if and only if $C_{y}(x)=0$.

- Since the straddling sets for the encodings for the input bits above use different sets $X_{i, b}$ for each input position and value, we need one more modification of the scheme to enable addition operations in the evaluated circuit. Since in the underlying graded encoding scheme we can add only encodings associated with the same level set, in order to enable addition of the values encoded in the signal slot in $\tilde{x}_{i, b}=\left[b, \alpha_{i}\right]_{X_{i, b}}$ and $\tilde{x}_{j, b^{\prime}}=\left[b^{\prime}, \alpha_{j}\right]_{X_{j, b^{\prime}}}$ the obfuscation provides also the following encodings: $\tilde{u}_{i, b}=[1,1]_{X_{i, b}}$ and $\tilde{u}_{j, b^{\prime}}=[1,1]_{X_{j, b^{\prime}}}$. The encoding of the sum of the signal slot values and the auxiliary encoding used for further additions are computed as

$$
\tilde{x}_{i, b} \tilde{u}_{j, b^{\prime}}+\tilde{x}_{j, b^{\prime}} \tilde{u}_{i, b}=\left[b+b^{\prime}, \alpha_{i}+\alpha_{j}\right]_{X_{i, b} X_{j, b^{\prime}}} \quad \tilde{u}_{i, b} \tilde{u}_{j, b^{\prime}}=[1,1]_{X_{i, b} X_{j, b^{\prime}}}
$$




\section{Attack Sets}

For our attack we will consider a circuit $C$ that has only multiplication gates and odd number $n$ of inputs. Our attack can be extended to "universal" circuits for point functions of the form $C(y, x)=C_{y}(x)=\chi_{x \oplus y=0}$. This circuit is described with a point $y$, it gets as input a point $x$, compares the respective bits of $x$ and $y$ and then computes an AND of the results. We can apply the attack that we describe next using the encoded bits $b_{i}=x_{i} \oplus y_{i}$ in the role of the input bits.

We split the $k$ bits of the input $x$ into three sets $X_{1}, X_{2}$ and $X_{3}$ where $X_{1}$ contains the first $(k-1) / 2$ bits, $X_{2}$ is the middle bit and $X_{3}$ are the last $(k-1) / 2$ bits of the input.

We construct the sets $\mathcal{A}, \mathcal{B}$ and $\mathcal{C}$ as follows:

- $\mathcal{A}=\left\{\prod_{i \in X_{1}}\left(\tilde{x}_{i, x_{i}} \tilde{z}_{i, x_{i}}\right)\right.$ : at least one of the values $\left\{\tilde{x}_{i, x_{i}}\right\}_{i \in X_{1}}$ encodes 0$\}$,

- $\mathcal{B}=\left\{b_{x_{(k-1) / 2+1}}=\tilde{x}_{(k-1) / 2+1, x_{(k-1) / 2+1}} \cdot \tilde{z}_{(k-1) / 2+1, x_{(k-1) / 2+1}}: x_{(k-1) / 2+1} \in\{0,1\}\right\}$,

- $\mathcal{C}=\left\{\prod_{i \in X_{3}}\left(\tilde{x}_{i, x_{i}} \tilde{z}_{i, x_{i}}\right): X_{3} \in\{0,1\}^{(k-1) / 2}\right\}$.

We have $s=1$ and $t=\mathbf{p}_{\mathrm{zt}}$. Also $M=b_{0} \times b_{1}^{-1}$.

Set Properties. Let $\mathcal{A}=\left\{a_{i}\right\}_{i \in[n+1]}, \mathcal{B}=\left\{b_{0}, b_{1}\right\}$ and $\mathcal{C}=\left\{c_{k}\right\}_{k \in[n+1]}$. Let $u=\tilde{C}^{*} \prod_{i \in[n]} \tilde{w}_{i, x_{i}}$. We consider

$$
W_{0}[j, k]=s \times a_{j} \times b_{0} \times c_{k} \times t-u \times t=g^{-1} h \cdot\left(u_{x_{1}} \cdot u_{x_{2}} \cdot u_{x_{3}}-u\right),
$$

where $g^{-1} h, u_{x_{1}}, u_{x_{2}}, u_{x_{3}}$ and $u$ have the following CRT components:

$$
\begin{aligned}
g^{-1} h & =\left(\begin{array}{lllll}
g_{1}^{-1} h_{1}, & g_{2}^{-1} h_{2}, & g_{3}^{-1} h_{3}, & \ldots, & g_{n}^{-1} h_{n}
\end{array}\right) \\
u_{x_{1}} & =\left(\begin{array}{lllll}
g_{1} \rho_{x_{1}, 1}, & g_{2} \rho_{x_{1}, 2}+r_{x_{1}}, & g_{3} \rho_{x_{1}, 3}, & \ldots, & g_{t} \rho_{x_{1}, n}
\end{array}\right) \\
u_{x_{2}} & =\left(\begin{array}{lllll}
\tau_{x_{2}, 1}, & g_{2} \tau_{x_{2}, 2}+r_{x_{2}}, & \tau_{x_{2}, 3}, & \ldots, & \tau_{x_{2}, n}
\end{array}\right) \\
u_{x_{3}} & =\left(\begin{array}{lllll}
\sigma_{x_{3}, 1}, & g_{2} \sigma_{x_{3}, 2}+r_{x_{3}}, & \sigma_{x_{3}, 3}, & \ldots, & \sigma_{x_{3}, n}
\end{array}\right) \\
u & =\left(\begin{array}{lllll}
g_{1} \psi_{1}, & g_{2} \psi_{2}+r^{*}, & g_{3} \psi_{3}, & \ldots, & g_{n} \psi_{n}
\end{array}\right) .
\end{aligned}
$$

The above CRT component correspond to CLT13 encoding of the value in the signal slot except the the values in the second CRT component which is the control slot. Since $u_{x_{1}}$ encodes 0 in its signal slot, all its CRT components are $0 \bmod p_{i}$ except the component in the second slot.

Let denote $\rho_{x_{1}, 2}^{\prime}=g_{2} \rho_{x_{1}, 2}+r_{x_{1}}, \tau_{y, 2}^{\prime}=g_{2} \tau_{x_{2}, 2}+r_{x_{2}}, \sigma_{z, 2}^{\prime}=g_{2} \sigma_{x_{3}, 2}+r_{x_{3}}$, and $\psi_{2}^{\prime}=g_{2} \psi_{2}+r^{*}$. Then, $W_{0}[j, k]$ equals

$g^{-1} h \cdot\left(g_{1}\left(\rho_{x_{1}, 1} \tau_{x_{2}, 1} \sigma_{x_{3}, 1}-\psi_{1}\right),\left(\rho_{x_{1}, 2}^{\prime} \tau_{x_{2}, 2}^{\prime} \sigma_{x_{3}, 2}^{\prime}-\psi_{2}^{\prime}\right), g_{3}\left(\rho_{x_{1}, 3} \tau_{x_{2}, 3} \sigma_{x_{3}, 3}-\psi_{3}\right), \ldots, g_{n}\left(\rho_{x_{1}, n} \tau_{x_{2}, n} \sigma_{x_{3}, n}-\psi_{n}\right)\right)$,

which is a zero-tested encoding of 0 since by construction $\psi_{2}^{\prime}-\rho_{x_{1}, 2}^{\prime} \tau_{x_{2}, 2}^{\prime} \sigma_{x_{3}, 2}^{\prime}\left(\bmod g_{2}\right)$. The above equality holds not only $\bmod x_{0}$ but also over the integers since $g^{-1}$ cancels out and all other variables are much smaller than $x_{0}$. 
We can write the last equality in matrix notations as

$$
\begin{aligned}
& W_{0}[j, k]=g^{-1} \cdot \overbrace{\left(\begin{array}{llllll}
g_{1} \rho_{x, 1} & \rho_{x_{1}, 2}^{\prime} & g_{3} \rho_{x_{1}, 3} & \ldots & g_{n} \rho_{x_{1}, n} & 1
\end{array}\right)}^{\mathbf{a}_{x_{1}}} \\
& \times \underbrace{\left(\begin{array}{cccccc}
h_{1} \tau_{x_{2}, 1} & & & & & \\
& h_{2} \tau_{x_{2}, 2}^{\prime} & & & & \\
& & h_{3} \tau_{x_{2}, 3} & & & \\
& & & \ddots & & \\
& & & & h_{n} \tau_{x_{2}, n} & \\
& & & & & -\psi^{*}
\end{array}\right)}_{B_{x_{2}}} \times \underbrace{\left(\begin{array}{c}
\sigma_{x_{3}, 1} \\
\sigma_{x_{3}, 2}^{\prime} \\
\sigma_{x_{3}, 3} \\
\vdots \\
\sigma_{x_{3}, n} \\
1
\end{array}\right)}_{\mathbf{c}_{x_{3}}}
\end{aligned}
$$

where $\psi^{*}=\psi_{2}^{\prime} h_{2}+\sum_{i \neq 2} g_{i} \psi_{i} h_{i}$, which depends only on $u, g$ and $h$ and is independent of the values in $\mathcal{B}$.

We construct matrix $A$ using $n+1$ vectors $\mathbf{a}_{x_{1}}$ obtained from the set $\mathcal{A}$ and matrix $C$ using $n+1$ vectors $\mathbf{c}_{x_{3}}$ vectors obtained from $\mathcal{C}$ as columns. We thus get the two matrices

$$
W_{0}=g^{-1} \cdot A \times B_{0} \times C \text { and } W_{1}=g^{-1} \cdot A \times B_{1} \times C,
$$

The matrices $W_{j}$ are invertible with high probability over the encodings' randomness by the same argument as in the attack on BWZ (Section 3.2. Hence $W=W_{0} \times W_{1}^{-1}=\tilde{A} \times \tilde{B}_{0} \times \tilde{B}_{1}^{-1} \times \tilde{A}^{-1}$, and

$$
\begin{aligned}
\operatorname{charPoly}(W)=\operatorname{charPoly}\left(\tilde{B}_{0} \times \tilde{B}_{1}^{-1}\right) & =\prod_{i=1}^{n}\left(\operatorname{charPoly}\left(b_{0} \times\left(b_{1}\right)^{-1} \bmod p_{i}\right)\right) \\
& =\prod_{i=1}^{n}\left(\operatorname{charPoly}\left(M \bmod p_{i}\right)\right),
\end{aligned}
$$

which is the first property for our attack sets.

The second through fourth properties of Definition 1 follow via essentially the same argument as in the attack on BWZ (Section 3.2).

\section{A Weak-DL Attack on Matrix-GGH13}

Given the GGH13 "weak discrete logarithm" attacks [10], a matrix variant of GGH13 can be constructed with the aim of avoiding public GGH13 encodings of zero in the public parameters (fashioned after the "Multilinear Jigsaw Puzzle" techniques in [11]). This variant replaces the native GGH13 encoding of the values of interest by encoding matrices related to these values (e.g. have the desired value as an eigenvalue). In Section 3.3, we have shown that a similar GGHZ countermeasure for CLT can be broken. In this section we show that the matrix GGH13 is also susceptible to a new "weak-discrete-logarithm" attack.

As in GGH13, we have plaintext space $R_{g}=R / g R$ where $R$ is the ring of integers in some number field and $g \in R$, and ciphertext space $R_{q}=R / q R$ for some integer $q$. Below we describe the attack in the "asymmetric" setting where we encode elements relative to subsets of $[k]=\{1, \ldots, k\}$, 
and use $[k]$ itself as our zero-test level. The secrets associated with an instance of the scheme include the plaintext-space element $g$ and $k$ random denominators $z_{i} \in R_{q}$.

Trying to protect against "zeroizing attacks", one could consider the following solution: we choose a random small vector $\mathbf{s}^{*} \in R^{n}$ and invertible matrix $T \in R_{q}^{n \times n}$ (for some $n$ ), and then encode $\alpha \in R_{g}$ relative to the set $S \subset[k]$ by choosing a random small matrix $A^{*}$ such that $\mathbf{s}^{*} \times A^{*}=\alpha \mathbf{s}^{*}(\bmod g R)$, and publishing the matrix

$$
A^{\{S\}}=\left[T \times A^{*} \times T^{-1} / \prod_{i \in S} z_{i}\right]_{q} .
$$

For the purpose of zero-test we choose another mid-size random vector $\mathbf{t}^{*} \in R^{n}$ and publish the two vectors $\mathbf{s}=\left[g^{-1} \mathbf{s}^{*} \times T^{-1}\right]_{q}$ and $\mathbf{t}=\left[\prod_{i} z_{i} \cdot T \times \mathbf{t}^{*}\right]_{q}$. (The $h$ element from GGH13 is implicitly defined as $h=\left\langle\mathbf{t}^{*}, \mathbf{s}^{*}\right\rangle$, which is indeed a mid-size element.) It is easy to see that this provides the functionality of a graded-encoding scheme, where a level- $[k]$ encoding $A^{[k]}$ can be tested for zero by checking that

$$
\left\|\left[\mathbf{s} \times A^{[k]} \times \mathbf{t}\right]_{q}\right\| \ll q .
$$

On the other hand, it seems hard to obtain a native GGH13-encoding of zero even if we are given matrices $A^{\{i\}}$ that encode zero, so we could naively hope that the zeroizing attacks from [10] do not apply. Unfortunately, we show that this is not the case.

\subsection{The Updated Weak-DL Attack.}

As in the original "weak-discrete-logarithm" attack from [10], we use encodings of "double zeros" in order to recover the ideal $I_{g}=g R$ and then use the knowledge of that ideal together with "single zeros" to attack other encodings. We use below the same "attack set" terminology as in our CLT attacks.

Attack Sets. As in all other attacks in the current work, we are given three sets of encodings, at levels corresponding to a partition of $[k]$ so that they product is the top level. Let $S_{1}, S_{2}, S_{3} \subset[k]$ be disjoint, and their union is $[k]$. Specifically assume that we are given the following encoding.

- Many level- $S_{1}$ encodings of zero

$$
\mathcal{A}^{S_{1}}=\left\{A_{j}^{S_{1}}=\left[T \times A_{j}^{*} \times T^{-1} / \prod_{i \in S_{1}} z_{i}\right]_{q}: \mathbf{s}^{*} A_{j}^{*}=\mathbf{0} \quad(\bmod g R)\right\}_{j}
$$

- Many level- $S_{2}$ encodings of zero and the two "target encodings" at level $S_{2}$ (encoding two random scalars $\delta_{0}, \delta_{1} \in R$ )

$$
\begin{aligned}
& \mathcal{Z}^{S_{2}}=\left\{Z_{j}^{S_{2}}=\left[T \times Z_{j}^{*} \times T^{-1} / \prod_{i \in S_{2}} z_{i}\right]_{q}: \mathbf{s}^{*} Z_{j}^{*}=\mathbf{0} \quad(\bmod g R)\right\}_{j} \\
& \mathcal{B}^{S_{2}}=\left\{B_{\sigma}^{S_{2}}=\left[T \times B_{\sigma}^{*} \times T^{-1} / \prod_{i \in S_{2}} z_{i}\right]_{q}\right\}_{\sigma=0,1},
\end{aligned}
$$


such that $\mathbf{s}^{*} B_{\sigma}^{*}=\delta_{\sigma} \mathbf{s}^{*}(\bmod g R)$ for $\sigma=0,1$, where the $\delta$ 's are small.

- Many level- $S_{3}$ encodings of nonzero elements

$$
\mathcal{C}^{S_{3}}=\left\{C_{j}^{S_{3}}=\left[T \times C_{j}^{*} \times T^{-1} / \prod_{i \in S_{3}} z_{i}\right]_{q}: \mathbf{s}^{*} C_{j}^{*}=\varepsilon_{j} \mathbf{s}^{*} \quad(\bmod g R)\right\}_{j} .
$$

for small scalars $\varepsilon_{j} \in R, \varepsilon_{j} \notin g R$.

Given these encodings, we show how to recover the ratio $\delta_{0} / \delta_{1}(\bmod g R)$. The set $\mathcal{Z}^{S_{2}}$ is only used to recover (a representation of) the ideal $I_{g}=g R$. Below we first assume that we know $I_{g}$ and show how to recover the ratio $\delta_{0} / \delta_{1}(\bmod g R)$, and later explain how $I_{g}$ can be recovered using $\mathcal{Z}^{S_{2}}$.

Observe that since the $A_{j}^{S_{1}}$ 's are encodings of zero then they cancel the term $g^{-1}$ in the vector s. Namely there exist small vectors $\mathbf{u}_{j} \in R^{n}$ such that $\mathbf{s}^{*} A_{j}^{*}=g \cdot \mathbf{u}_{j}$, and therefore $\left[\prod_{i \in S_{1}} z_{i} \cdot \mathbf{s} \times A_{j}^{S_{1}} \times T\right]_{q}=\mathbf{s}^{*} A_{j}^{*} / g=\mathbf{u}_{j}$. Let us also denote below $\mathbf{v}_{j}=\left[\prod_{i \in S_{3}} z_{i} \cdot T \times C_{j}^{S} \times \mathbf{t}\right]_{q}$ and notice $\mathbf{v}_{j}=C_{j}^{S, *} \mathbf{t}^{*}$ (equality in $R$ ) and $\mathbf{v}_{j}$ is the small. Then for all $i, \sigma, j$ we get

$$
W_{\sigma}[i, j]:=\left[\mathbf{s} \times A_{i}^{S_{1}} \times B_{\sigma}^{S_{2}} \times C_{j}^{S_{3}} \times \mathbf{t}\right]_{q}=\mathbf{u}_{i} \times B_{\sigma}^{*} \times \mathbf{v}_{j}(\text { equalities over } R) .
$$

Denote by $U, V \in R^{n \times n}$ the matrices with $\mathbf{u}_{j}$ 's as rows and $\mathbf{v}_{j}$ 's as columns, respectively, and also define the two $n \times n$ matrices $W_{\sigma}=W_{\sigma}[i, j]_{i, j}$. Then by definition we have $W_{\sigma}=U \times B_{\sigma}^{*} \times V$ for $\sigma=0,1$.

Assuming that we know the plaintext space ideal $I_{g}$ and that $W_{1}$ is invertible modulo $I_{g}$, we can proceed similarly to the CLT attacks by computing the eigenvalues of $W=W_{0} \times W_{1}^{-1}$, but this time modulo $I_{g}$, and noting that the ratio $\delta_{0} / \delta_{1}$ must be one of these eigenvalues. (If $W_{1}$ is not invertible modulo $I_{g}$, we can still recover the same by finding the roots of the polynomial $p(\lambda)=\lambda W_{1}-W_{0}$.) This implies that as long as we know the ideal $I_{g}$ (and can find roots of polynomials modulo $I_{g}$ ), then similarly to the original "weak-discrete-log" attack from [10] we can recover any encoded value $\delta$ at level $S_{2}$ up to a multiplicative factor of $1 / \delta_{1}$.

If we are not explicitly given the ideal $I_{g}$, then we can use the set $\mathcal{Z}^{S_{2}}$ to recover it. Similarly to above we define

$$
X_{\ell}[i, j]:=\left[\mathbf{s} \times A_{i}^{S_{1}} \times Z_{\ell}^{S_{2}} \times C_{j}^{S_{3}} \times \mathbf{t}\right]_{q}=\mathbf{u}_{i} \times Z_{\ell}^{*} \times \mathbf{v}_{j}
$$

and then we have $X_{\ell}=X_{\ell}[i, j]_{i, j}=U \times Z_{\ell}^{*} \times V$, with equalities over $R$

Computing the determinant of all the matrices $W_{\sigma}$ and $X_{\ell}$, we observe that they are all divisible by $\operatorname{det}(U V)$, and with good probability this is the only common factor. We can therefore take the GCD (over the ideals) to recover and eliminate the factor $\operatorname{det}(U V)$, thereby getting the ideals that are generated by all the elements $t_{\ell}:=\operatorname{det}\left(Z_{\ell}^{*}\right)$. Recalling that all the $Z_{\ell}^{*}$ 's are singular modulo $I_{g}$ (since $\mathbf{s}^{*} \times Z_{\ell}^{*} \in g R$ ), we have that $g$ divides all the $t_{\ell}$ 's, and again w.h.p. this is the only factor that divides them all. We therefore can use GCD again to recover the ideal $I_{g}$ from all the ideals $I_{t_{\ell}}=t_{\ell} R$. 


\section{Conclusions}

In this work we extended the recent CHLRS zeroizing attacks to many new settings, and also illustrated some of the limitations of this attack technique. The underlying message of recent attacks is that for current multilinear-map candidates, successful zero-tests give the adversary equations over the base ring (i.e. the integers or the ring of integers in a number field). Understanding the security of these candidates therefore hinges on a better understanding of which types of systems of nonlinear equations can be solved efficiently.

\section{References}

[1] B. Applebaum and Z. Brakerski. Obfuscating circuits via composite-order graded encoding. In Y. Dodis and J. B. Nielsen, editors, Theory of Cryptography - TCC'15, Part II, volume 9015 of Lecture Notes in Computer Science, pages 528-556. Springer, 2015. http://eprint.iacr. org/2015/025.

[2] S. Badrinarayanan, E. Miles, A. Sahai, and M. Zhandry. Post-zeroizing obfuscation: The case of evasive circuits. Cryptology ePrint Archive, Report 2015/167, 2015. http://eprint.iacr. org/.

[3] B. Barak, S. Garg, Y. T. Kalai, O. Paneth, and A. Sahai. Protecting obfuscation against algebraic attacks. In P. Q. Nguyen and E. Oswald, editors, EUROCRYPT 2014, volume 8441 of Lecture Notes in Computer Science, pages 221-238. Springer, 2014.

[4] D. Boneh, D. J. Wu, and J. Zimmerman. Immunizing multilinear maps against zeroizing attacks. Cryptology ePrint Archive, Report 2014/930, 2014. http://eprint.iacr.org/.

[5] Z. Brakerski and G. N. Rothblum. Virtual black-box obfuscation for all circuits via generic graded encoding. In Y. Lindell, editor, Theory of Cryptography - TCC 2014, volume 8349 of Lecture Notes in Computer Science, pages 1-25. Springer, 2014.

[6] J. H. Cheon, K. Han, C. Lee, H. Ryu, and D. Stehlé. Cryptanalysis of the multilinear map over the integers. In E. Oswald and M. Fischlin, editors, Advances in Cryptology - EUROCRYPT 2015, Part I, volume 9056 of Lecture Notes in Computer Science, pages 3-12. Springer, 2015. http://eprint.iacr.org/2014/906.

[7] J. Coron, T. Lepoint, and M. Tibouchi. Practical multilinear maps over the integers. In R. Canetti and J. A. Garay, editors, CRYPTO 2013, volume 8042 of Lecture Notes in Computer Science, pages 476-493. Springer, 2013.

[8] J. Coron, T. Lepoint, and M. Tibouchi. Cryptanalysis of two candidate fixes of multilinear maps over the integers. Cryptology ePrint Archive, Report 2014/975, 2014. http://eprint. iacr.org/.

[9] J. Coron, T. Lepoint, and M. Tibouchi. New multilinear maps over the integers. In R. Gennaro and M. Robshaw, editors, CRYPTO 2015, Lecture Notes in Computer Science. Springer, 2015. To appear. 
[10] S. Garg, C. Gentry, and S. Halevi. Candidate multilinear maps from ideal lattices. In T. Johansson and P. Q. Nguyen, editors, EUROCRYPT 2013, volume 7881 of Lecture Notes in Computer Science, pages 1-17. Springer, 2013.

[11] S. Garg, C. Gentry, S. Halevi, M. Raykova, A. Sahai, and B. Waters. Candidate indistinguishability obfuscation and functional encryption for all circuits. In FOCS 2013, pages 40-49. IEEE Computer Society, 2013.

[12] S. Garg, C. Gentry, S. Halevi, and M. Zhandry. Fully secure functional encryption without obfuscation. Cryptology ePrint Archive, Report 2014/666, 2014. http://eprint.iacr.org/

[13] C. Gentry, S. Halevi, H. K. Maji, and A. Sahai. Zeroizing without zeroes: Cryptanalyzing multilinear maps without encodings of zero. Cryptology ePrint Archive, Report 2014/929, 2014. http://eprint.iacr.org/

[14] C. Gentry, A. B. Lewko, A. Sahai, and B. Waters. Indistinguishability obfuscation from the multilinear subgroup elimination assumption. IACR Cryptology ePrint Archive, 2014:309, 2014 .

[15] C. Gentry, A. B. Lewko, and B. Waters. Witness encryption from instance independent assumptions. In J. A. Garay and R. Gennaro, editors, CRYPTO 2014, volume 8616 of Lecture Notes in Computer Science, pages 426-443. Springer, 2014.

[16] S. Goldwasser and S. Micali. Probabilistic encryption. J. Comput. Syst. Sci., 28(2):270-299, 1984.

[17] J. C. Hansen and E. Schmutz. How random is the characteristic polynomial of a random matrix? Math. Proc. Camb. Phi. Soc., 114:507-515, 1993.

[18] Y. Hu and H. Jia. Cryptanalysis of GGH map. Cryptology ePrint Archive, Report 2015/301, 2015. http://eprint.iacr.org/

[19] G. Kuba. On the distribution of reducible polynomials. Math. Slovaca, 59(3):349-356, 2009.

[20] E. Miles, A. Sahai, and M. Weiss. Protecting obfuscation against arithmetic attacks. IACR Cryptology ePrint Archive, 2014:878, 2014.

[21] R. Pass, K. Seth, and S. Telang. Indistinguishability obfuscation from semantically-secure multilinear encodings. In CRYPTO 2014, pages 500-517, 2014.

[22] J. Zimmerman. How to obfuscate programs directly. In E. Oswald and M. Fischlin, editors, Advances in Cryptology - EUROCRYPT 2015, Part II, volume 9057 of Lecture Notes in Computer Science, pages 439-467. Springer, 2015. http://eprint.iacr.org/2014/776.

\section{A A Refined Generic Model}

The zeroising attacks from [10, 6] and the current work point to the inadequacy of the generic graded-encoding model as used in recent work. Indeed these attacks are highly algebraic and yet 
they are not captured by that generic model. The main difference is that in the generic gradedencoding model the zero-test returns just a 0/1 bit, whereas in the GGH13/CLT13 schemes this test returns a full ring element.

We therefore propose to augment this generic model as follows: In addition to the standard interfaces in the graded-encoding model (with some plaintext space $R^{\prime}=R / g R$ ), we will now also have a black-box-field over the ring $R$, except that we cannot directly obtain handles to this blackbox field. Instead, the zero-test would serve as a translation device, letting us move things from the graded-encoding oracle to the black-box-field oracle.

In more detail, we would have the usual oracles to sample/encode elements in the gradedencoding scheme and to add and multiply them with the usual semantics. However, with each encoded element the graded-encoding oracle will also associate a "random element of $R$ " from the appropriate coset. Namely, with each encoded value $\alpha$ the oracle will also have an associated $r_{\alpha} \in R$ and the intended semantics is that we use $\alpha+g \cdot r_{\alpha}$ to represent the coset $\alpha+g R$. The oracle keeps track of the representatives via the addition and multiplication operations of the graded-encoding scheme, by adding and multiplying the representatives in the ring $R$.

Then, if the zero-test is called on an encoding of zero with representative $g \cdot r \in R$, then in addition to the bit 1 the oracle will also give us a handle to the element $r \in R$ in the black-box field. Namely, if we call it on an element which is divisible by $g$ then it will divide by $g$ and move it to the black-box field.

\section{B Hardness Assumption with Binding Variables}

Below we describe a relatively simple source-group hardness assumption involving "binding variables", similar to thte ones used, e.g., in [3, 22]. In this assumption we are given many low-level CLT13 encodings of zero, and yet we do not know how to break it. We stress that this assumption is not meant to be useful for any crypto applications, its goal is just to highlight the difficulty that such binding variables pose when trying to mount a zeroizing attack.

This assumption is staged in the "asymmetric multilinear setting" where the levels correspond to integer vectors rather than to simple integers. (This setting makes it more convenient to enforce the need to multiply by the binding variables.) It would be interesting either to show how to extend the attacks so as to break this hardness assumption, or to provide some evidence that this line of attacks is incapable of breaking it.

The setting in this hardness assumption consists of $4\left(\begin{array}{l}n \\ 2\end{array}\right)+2 n+1$ encoded variables: One is the target encoding, which is either a zero encoding $U^{*} \sim\left(g_{i} r_{i}^{*}, \ldots, g_{n} r_{n}^{*}\right)$ or an encoding of a random vector $U^{*} \sim\left(r_{1}^{*}, \ldots, r_{n}^{*}\right)$. There are $4\left(\begin{array}{c}n \\ 2\end{array}\right)$ binding variables $U_{i, j, \sigma_{1}, \sigma_{2}}$ and $2 n$ filler variables $V_{i, \sigma}$, all of them encoding zeros. That is, $U_{i, j, \sigma_{1}, \sigma_{2}} \sim\left(g_{1} r_{i, j, \sigma_{1}, \sigma_{2}}^{1}, \ldots, g_{n} r_{i, j, \sigma_{1}, \sigma_{2}}^{n}\right)$ and $V_{i, \sigma} \sim\left(g_{1} r_{i, \sigma}^{1}, \ldots, g_{n} r_{i, \sigma}^{n}\right)$.

The levels of these encodings are designed to ensure that the only way to get an encoding of zero at the top level is multiply together the target encoding $U^{*}$ and other variables that are consistent with some $n$-bit string $x$. Namely, the $n$ filler encodings $V_{i, x_{i}}, i=1, \ldots, n$, and the $\left(\begin{array}{l}n \\ 2\end{array}\right)$ biding encodings $U_{i, j, x_{i}, x_{j}}, 1 \leqslant i<j \leqslant n$. The level structure of the scheme consists of integer vectors of dimension $1+2 n+\left(\begin{array}{l}n \\ 2\end{array}\right)$, as described below. In this description we let $e_{i}$ denote the $i$ 'th unit vector, with indexing beginning at zero.

- The target variable $U^{*}$ is encoded relative to level $e_{0}=(1,0, \ldots, 0)$. 
- The filler variable $V_{i, 0}$ is encoded relative to level $(n-1) \cdot e_{i}$ (i.e. $n-1$ in position $i$ and 0 elsewhere), and the filler variable $V_{i, 1}$ is encoded relative to level $(n-1) \cdot e_{i+n}$.

- Let $k$ be the index of the pair $(i, j)$. Then the levels of all four binding variable $U_{i, j, *, *}$ 's have 1 in position $k$ and also 1's in two of the four positions $i, j, n+i, n+j$ :

- $U_{i, j, 0,0}$ is encoded at level $e_{k}+e_{n+i}+e_{n+j}$;

- $U_{i, j, 1,0}$ is encoded at level $e_{k}+e_{i}+e_{n+j}$;

- $U_{i, j, 0,1}$ is encoded at level $e_{k}+e_{n+i}+e_{j}$; and

- $U_{i, j, 1,1}$ is encoded at level $e_{k}+e_{i}+e_{j}$.

- The top level (where we perform zero-test) corresponds to the vector

$$
e_{0}+(n-1)\left(\sum_{k=1}^{2 n} e_{k}\right)+\sum_{k=1}^{\left(\begin{array}{c}
n \\
2
\end{array}\right)} e_{2 n+k}=(1, \underbrace{n-1, \ldots, n-1}_{2 n \text { entries }}, \underbrace{1, \ldots, 1}_{\left(\begin{array}{c}
n \\
2
\end{array}\right) \text { entries }}) .
$$

Recall that multiplying encodings add their levels (as vectors over the integers). So we want to show that the only way to hit the target level is by adding levels of the input encodings in a manner consistent with some $n$-bit string $x$. Clearly we need to take one from every four $U_{i, j, *, *}$ 's to get all the ones in the last $\left(\begin{array}{l}n \\ 2\end{array}\right)$ positions. These $\left(\begin{array}{l}n \\ 2\end{array}\right)$ vectors contributes a "weight" of $2\left(\begin{array}{l}n \\ 2\end{array}\right)=n(n-1)$ in positions $1, \ldots, n$, so we need to add $n$ more fillers to make up the missing "weight" of $n(n-1)$ in these positions.

But we cannot add $V_{i, 0}$ if for some $j$ we used $u_{i, j, 1, *}$, and similarly cannot add $V_{i, 1}$ if for some $j$ we used $u_{i, j, 0, *}$ (lest we overshoot the $n-1$ target in positions $i$ or $n+i$ ). Hence it must be the case that for every $i$ we used exactly one of $V_{i, 0}, V_{i, 1}$, and for all $i, j$ if we used $V_{i, \sigma_{1}}$ and $V_{j, \sigma_{2}}$ then we also use $U_{i, j, \sigma_{1}, \sigma_{2}}$.

\section{B.1 Attacking Simplified Binding Variables}

The reason that binding variables make it hard to mount zeroizing attacks is that for every input $t=x y z$ that leads to zero, we no longer get the nice separated expression $w_{t}=\mathbf{a}_{x} \times B_{y} \times \mathbf{c}_{z}$ as before, but rather it is multiplied by these variables. Namely rather than the four-wise innerproduct that we use in the attacks in the work, we now have many more vectors that come from the binding variables, and we cannot partition these vectors in a nice way.

Below we show, however, that in a simplified setting where these vectors are replaced by individual integers, we can use GCD computations to remove them and then mount the standard zeroizing attacks. Assume that our zero-tested top-level zeros are given by the following expression:

$$
w_{t}=\mathbf{a}_{x} \times B_{y} \times \mathbf{c}_{z} \cdot \prod_{i<j} u_{i, j, t_{i}, t_{j}} .
$$

Let $X, Y, Z$ denote the bit positions belonging to the $x, y, z$ parts of the input, respectively, and consider a set of inputs that vary $x, z$ and keep $y$ fixed, $t^{k}=x^{k} y z^{k}$. Since $y$ is fixed then is means that for $i, j \in Y$ we always get the same two bit values for $t_{i}^{k}, t_{j}^{k}$ (for all $k$ ). But for $(i, j) \in X \times Y$ and for $(i, j) \in Y \times Z$ we can have two different values for the pair $\left(t_{i}^{k}, t_{j}^{k}\right)$ for different $k$ 's, and 
for $(i, j) \in X \times X,(i, j) \in X \times Z$, and $(i, j) \in Z \times Z$ we can have all four values. Assume that our collection of inputs is such that for all $i, j$ we have as much variability in the values $\left(t_{i}^{k}, t_{j}^{k}\right)$ as possible (subject to $y$ being fixed).

Also assume for simplicity that these binding variable are chosen to that for every $i, j$, the four variables $u_{i, j, b_{1}, b_{2}}$ are pairwise co-prime. Then all the $w_{t^{k}}$ 's have a factor of $U_{y}=\prod_{i, j \in Y} u_{i, j, y_{i}, y_{j}}$, but they are very unlikely to have any other common factors. Taking the GCD of all these $w_{t^{k}}$ 's can therefore give us $U_{y}$.

Assuming that we can have such sets of inputs for every desirable partition of the input bits, we would be able to compute the product $U_{Y}$ for every subset $Y \in[n]$. In particular we can compute it for every pair $\{i, j\} \subset[n]$, thereby recovering all the variables $u_{i, j, b_{1}, b_{2}}$. Then we can go back to Eqn. (1) and divide out these variables, thus recovering our simpler form and then running the attacks from above. 